\title{
Ion Coordination and Chelation in a Glycolated Polymer Semiconductor: Molecular Dynamics and X-Ray Fluorescence Study
}

Micaela Matta, Ruiheng Wu, Bryan D. Paulsen, Anthony Petty, Rajendar Sheelamanthula, lain McCulloch, George C Schatz, Jonathan Rivnay

Submitted date: 08/05/2020 - Posted date: 12/05/2020

Licence: CC BY-NC-ND 4.0

Citation information: Matta, Micaela; Wu, Ruiheng; Paulsen, Bryan D.; Petty, Anthony; Sheelamanthula, Rajendar; McCulloch, lain; et al. (2020): Ion Coordination and Chelation in a Glycolated Polymer Semiconductor: Molecular Dynamics and X-Ray Fluorescence Study. ChemRxiv. Preprint. https://doi.org/10.26434/chemrxiv.12264308.v2

Organic electrochemical transistors (OECTs) are based on the doping of a semiconducting polymer by an electrolyte. Due to their ability to conjugate volumetric ion penetration with high hole mobility and charge density, polythiophenes bearing glycolated side chains have rapidly surged as the highest performing materials for OECTs; amongst them, p(g2T-TT) is amongst those with the highest figure of merit. While recent studies have shown how different doping anions tend to affect the polymer microstructure, only a handful of electrolytes have been tested in mixed conduction devices. Our work provides an atomistic picture of the $\mathrm{p}(\mathrm{g} 2 \mathrm{~T}-\mathrm{TT})$-electrolyte interface in the 'off' state of an OECT, expected to be dominated by cation-polymer interactions. Using a combination of molecular dynamics simulations and X-ray fluorescence, we show how different anions effectively tune the coordination and chelation of cations by glycolated polymers. At the same time, softer and hydrophobic anions such as TFSI and $\mathrm{ClO}_{4}$ are found to preferentially interact with the $\mathrm{p}(\mathrm{g} 2 \mathrm{~T}-\mathrm{TT})$ phase, further enhancing polymer-cation coordination. Besides opening the way for a full study of electrolyte doping mechanisms in operating devices, our results suggest that tailoring the electrolyte for different applications and materials might be a viable strategy to tune the performance of mixed conducting devices.

File list (3)

g2TTT_Matta_chemrxiv.pdf (6.07 MiB)

view on ChemRxiv - download file

ionscape_text.pdf (1.14 MiB)

view on ChemRxiv • download file

SI_g2TTT_Matta.pdf (1.70 MiB)

view on ChemRxiv • download file 


\title{
Ion Coordination and Chelation in a Glycolated Polymer Semiconductor: Molecular Dynamics and X-Ray Fluorescence Study
}

Micaela Matta, ${ }^{1,2 *}$ Ruiheng $\mathrm{Wu},{ }^{3}$ Bryan D. Paulsen, ${ }^{3}$ Anthony J. Petty $\mathrm{II}^{3}{ }^{3}$ Rajendar Sheelamanthula, ${ }^{4}$ Iain McCulloch, ${ }^{4,5}$ George C. Schatz, ${ }^{1}$ Jonathan Rivnay ${ }^{3}$

${ }^{1}$ Northwestern University, Department of Chemistry, 2145 Sheridan Rd, Evanston, IL 60208, USA

${ }^{2}$ University of Liverpool, Department of Chemistry, Crown St., Liverpool L69 7ZD, United Kingdom

${ }^{3}$ Northwestern University, Department of Biomedical Engineering, 2145 Sheridan Rd, Evanston, IL 60208, USA

${ }^{4}$ King Abdullah University of Science and Technology (KAUST), KAUST Solar Center (KSC), Thuwal 23955-6900, Saudi Arabia

${ }^{5}$ Imperial College London, Department of Chemistry and Center for Plastic Electronics, London, W12 0BZ, United Kingdom

*micaela.matta@liverpool.ac.uk

University of Liverpool, Department of Chemistry, Crown st., Liverpool L69 7ZD, United Kingdom

\begin{abstract}
Organic electrochemical transistors (OECTs) are based on the doping of a semiconducting polymer by an electrolyte. Due to their ability to conjugate volumetric ion penetration with high hole mobility and charge density, polythiophenes bearing glycolated side chains have rapidly surged as the highest performing materials for OECTs; amongst them, p(g2T-TT) is amongst those with the highest figure of merit. While recent studies have shown how different doping anions tend to affect the polymer microstructure, only a handful of electrolytes have been tested in mixed
\end{abstract}


conduction devices. Our work provides an atomistic picture of the p(g2T-TT) -electrolyte interface in the 'off' state of an OECT, expected to be dominated by cation-polymer interactions. Using a combination of molecular dynamics simulations and X-ray fluorescence, we show how different anions effectively tune the coordination and chelation of cations by glycolated polymers. At the same time, softer and hydrophobic anions such as TFSI and $\mathrm{ClO}_{4}$ are found to preferentially interact with the $\mathrm{p}(\mathrm{g} 2 \mathrm{~T}-\mathrm{TT})$ phase, further enhancing polymer-cation coordination. Besides opening the way for a full study of electrolyte doping mechanisms in operating devices, our results suggest that tailoring the electrolyte for different applications and materials might be a viable strategy to tune the performance of mixed conducting devices.

\section{Introduction}

Organic electrochemical transistors (OECTs) are ionic-to-electronic transducers that rely on the ability of the organic active layer to transport both ions and electrons. These devices consist of an aqueous electrolyte in direct contact with a $\pi$-conjugated polymer channel. Upon an applied voltage, the ions are driven inside the polymer film (doping) and compensate excess mobile electronic charge density on the polymer backbone. This electrolyte gating results in lower operating voltages with respect to traditional field-effect transistors. Further, in OECTs the ion permeation involves the whole volume of the active layer yielding high signal amplification, but often slower response times. ${ }^{1}$

The great interest in OECTs and, more broadly, in mixed transport is proportional to the variety of applications where these devices have disrupted the state-of-the-art: from bioelectronics ${ }^{2,3}$ and healthcare (neural interfaces, chemical and biological sensors ${ }^{4,5}$ ), to energy production ${ }^{6}$ and storage, ${ }^{7}$ to artificial synapses. ${ }^{8,9}$ These applications exploit the similarity between OECT operation and the way our cells send and receive signals, opening facile integration with biological substrates.

At the heart of OECTs working principle is the active material, which is usually a $\pi$-conjugated polymer or polymer blend able to host or be chemically linked to charged groups. ${ }^{10}$ The greatest challenges of developing organic mixed ionic-electronic conductors (OMIECs) is the need to optimize the seemingly contrasting processes of electronic and ionic charge transport: while the 
factors governing electronic charge transport in conjugated polymers is now fairly well understood, ${ }^{11-13}$ little is known about how the presence of water and ions affects both the microstructure of the materials and its transport characteristics. On the other hand, although ion transport has been extensively studied in the context of capacitors and batteries, where it is one of the main governing processes, a systematic understanding of ion percolation in OMIECs is still in its infancy and limited to a few widespread materials.

Currently, the most employed material for OECTs is the commercially available PEDOT:PSS, that combines the semiconductor PEDOT with the polyelectrolyte PSS. Despite the good device characteristics (such as high transconductance and switching frequency) and a demonstrated biocompatibility, ${ }^{14,15}$ there is significant room for design and systematic improvement of polymer semiconductors for OECTs. ${ }^{1}$ In recent years, the strategy of swapping the solubilizing alkyl chains of $\pi$-conjugated polythiophenes in favor of ethylene glycol (EG) side chains has proven a promising strategy to achieve a balance between water permeability, ion mobility and hole transport efficiency. Amongst these glycolated mixed conductors, p(g2T-TT) (Figure 1a) demonstrated the best overall figure of merit, ${ }^{16}$ achieving high currents at submillisecond time scales, high transconductance, and steep subthreshold switching. ${ }^{17,18}$

However, despite the increasing library of OMIECs being tested in devices, comprehensive relationships between chemical modification, molecular and nanoscale morphology, extent of ion penetration and its impact on device characteristics are still largely missing. For this reason, a few studies aimed at elucidating the mechanism of ion conduction and ion uptake in hydrated $\mathrm{p}(\mathrm{g} 2 \mathrm{~T}$ TT) films, ${ }^{19,20}$ as well as other glycolated polythiophenes, have been carried out. It has been observed that the polymer undergoes swelling when in contact with the electrolyte solution (passive uptake), and again when bias is applied (active uptake). The polymer swelling was found to depend on the ion size and the nature of the electrolyte, however the mass uptake from passive swelling remains around 10\% (unlike for PEDOT:PSS where it can exceed 100\%). ${ }^{21,22}$ It appears that the changes in $\pi-\pi$ stacking and lamellar spacing upon doping are permanent, and the film microstructure is altered after the first doping cycle. This however does not affect performance, and the device operation is stable. This information suggests that some of the ions could be responsible for this 'structural doping' and might be strongly interacting with the EG side chains. ${ }^{19}$ 
It should be noted that such systems likely exhibit a low degree of crystallinity, and since it has been shown that the presence of disordered domains is crucial for ion mobility, ${ }^{23}$ the available structural data - which only represent crystalline domains - do not provide a complete picture of how ions interact with $\mathrm{p}(\mathrm{g} 2 \mathrm{~T}-\mathrm{TT})$. Notably, swelling and ion uptake measurements of thin films can only offer an average estimate, since water penetration and swelling are expected to be strongly dependent on the local environment in a material characterized by a rich microstructure. For this reason, molecular dynamics (MD) simulations can prove a valuable tool to zoom in at the molecular-scale and probe, separately, polymer-electrolyte interfaces with different degrees of order.

Perhaps one of the crucial understudied aspects regarding the microscopic behavior of OMIECs in devices is how the concentration and nature of the electrolyte affects OECT response and ultimately drives changes in the polymer microstructure. It is understood that during passive swelling cations interact with the polymer side chains, while upon doping the excess anions penetrating the polymer microstructure are expected to be more closely interacting with the polymer backbone, stabilizing polarons. In this respect, studies relating ion concentration, size and water uptake to the polymer morphology and performance have recently been reported; ${ }^{19,20,24}$ however, only very few electrolytes have been investigated.

In this study, we use molecular dynamics simulations and X-ray fluorescence spectroscopy to study p(g2T-TT)-electrolyte interfaces and obtain atomistic insight on polymer-electrolyte interactions. We investigate various salts, showing how moving along the Hofmeister series ${ }^{25}$ affects the polymer-electrolyte interface. In particular, we show how different anions compete with the EG side chains for cation coordination and chelation. Our study is focused on the interfacial interactions between a pure polymer phase and an electrolyte phase; we have not attempted here to simulate the entire swelling process since, as we stated above, it is not straightforward to estimate the degree of swelling in polymer regions having different microstructure. Furthermore, the microsecond time scale accessible within classical MD simulations is not sufficient to observe polymer swelling.

This study elucidates the impact of the electrolyte on the polymer film interfaces, and can be extended to the general class of $\pi$-conjugated polymers with EG side chains. Our simulations are 
performed in neutral conditions that mimic the 'off' state of an OECT, which undergoes passive swelling and electrolyte uptake. In general, our work is a necessary step towards the simulation of operando conditions, which would involve the presence of excess charges on the polymer backbone and an excess of anions, and further insight on the mutual interaction between them. A full description of the interaction between electronic and ionic charge carriers in a realistic environment is however beyond the current state of the art.

In the following three sections we present results arising from our computational study of the $\mathrm{p}(\mathrm{g} 2 \mathrm{~T}-\mathrm{TT})$-water interface. We initially focus on the trends in cation-polymer coordination in different electrolytes as a function of their ionic radius, hydration sphere and water structuring properties. We then analyze the differences in polymer-water hydrogen bonds and polymer surface area as a function of the electrolyte. Next, we show the density and electrostatic potential profiles of the polymer-electrolyte interface, focusing on interactions between hydrophobic anions and $\mathrm{p}(\mathrm{g} 2 \mathrm{~T}-\mathrm{TT})$. In the fourth section, we discuss the X-ray fluorescence study of $\mathrm{p}(\mathrm{g} 2 \mathrm{~T}-\mathrm{TT})$ thin films soaked in various electrolytes; these measurements are interpreted using insight from the MD simulations, providing validation of the anion-enabled chelation observed in silico.

\section{Results and Discussion}

A planar interface between $\mathrm{p}(\mathrm{g} 2 \mathrm{~T}-\mathrm{TT})$, water and various electrolytes (see Figure 1a) was built by interfacing a previously equilibrated bulk polymer periodic box and a water/electrolyte box along the $\mathrm{z}$ axis of the simulation cell (see Computational Methods for details). The polymer layer was obtained by annealing and equilibrating 20 coiled polymer bundles made of 16 repeating units each, to form a compact 2D film characterized by having the conjugated backbone $\pi$-stacking roughly parallel to the film plane, which could be described as an edge-on orientation. This entails that most of the solvent-exposed surface area is formed by side chains, while the backbone is largely buried inside the film (see Figure 1b and Computational Methods). 3D periodic boundary conditions apply, with the water-polymer interface along the $\mathrm{z}$ axis, and the pure polymer/pure water phases continuous along $\mathrm{x}$ and $\mathrm{y}$. All electrolytes were added in $1 \mathrm{M}$ concentration. In the case of $\mathrm{RbClO}_{4}$, the formation of ionic clusters was observed during simulations carried out at $1 \mathrm{M}$ 
concentration. This phenomenon has been previously observed for $\mathrm{ClO}_{4}$ salts. ${ }^{26}$ To prevent this, the simulation was carried out in $0.5 \mathrm{M} \mathrm{RbClO}_{4}$, where the formation of clusters was not observed.

a)

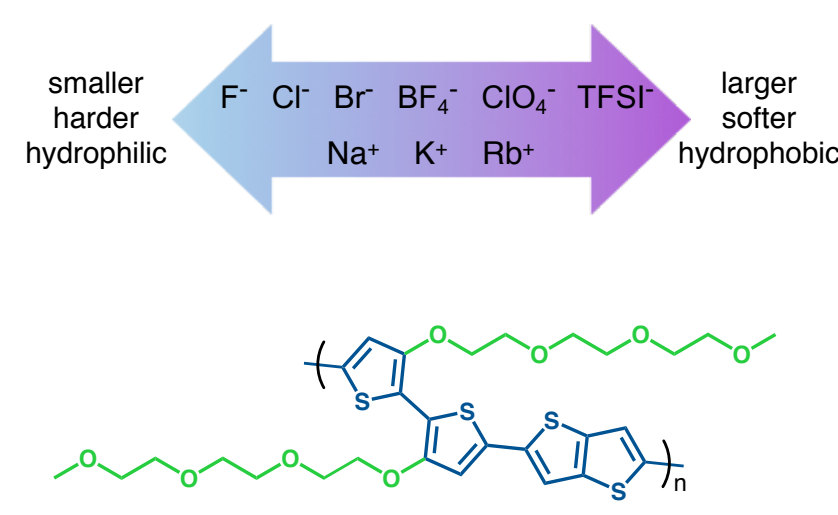

b)

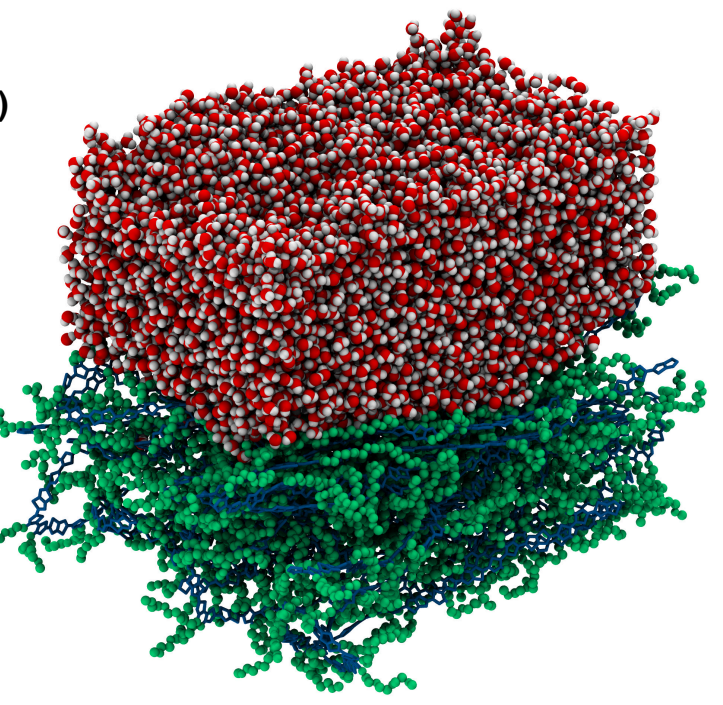

Figure 1. a) Scheme showing the anions and cations studied in this work as a function of their characteristics, as well as the chemical structure of $\mathrm{p}(\mathrm{g} 2 \mathrm{~T}-\mathrm{TT})$. b) Detail of the simulated interface between $\mathrm{p}(\mathrm{g} 2 \mathrm{~T}-\mathrm{TT})$ and water. Side chains are shown as green spheres while the polymer backbone is shown as blue lines. 3D Periodic boundary conditions apply.

\section{Coordination and chelation of cations by p(g2T-TT) side chains}

In this section, we analyze how water and $\mathrm{p}(\mathrm{g} 2 \mathrm{~T}-\mathrm{TT})$ compete for the coordination environment of $\mathrm{Na}^{+}, \mathrm{K}^{+}$and $\mathrm{Rb}^{+}$; we then investigate how the nature of the counterion modulates these interactions. Figure 2 shows the radial distribution function (RDF) of each metal ion with respect to its counterion, EG oxygens and water. As expected, going down the alkali metals group, cationanion interactions start to dominate the first solvation shell. In the same way, the outer solvation shell is increasingly less structured for heavier cations, whose influence on the water ordering does not extend beyond the first solvation layer. The same effect is seen when swapping harder anions for larger, more hydrophobic ones: $\mathrm{BF}_{4}^{-}, \mathrm{ClO}_{4}{ }^{-}$and $\mathrm{TFSI}^{-}$can penetrate the first solvation shell of $\mathrm{Na}^{+}$more effectively than halogens. Interestingly, TFSI- salts show lower RDF peaks with respect to other polyatomic anions (Figure 2a). These changes are accompanied by an increase in the metal-polymer peaks, denoting cation coordination to EG side chains at the expense of water which becomes less tightly bound; this is particularly evident if TFSI $^{-}$is used as a counterion (Figure 
2b). Overall, both the size/hardness of anions and cations play a role in tuning the extent of cationpolymer interactions.
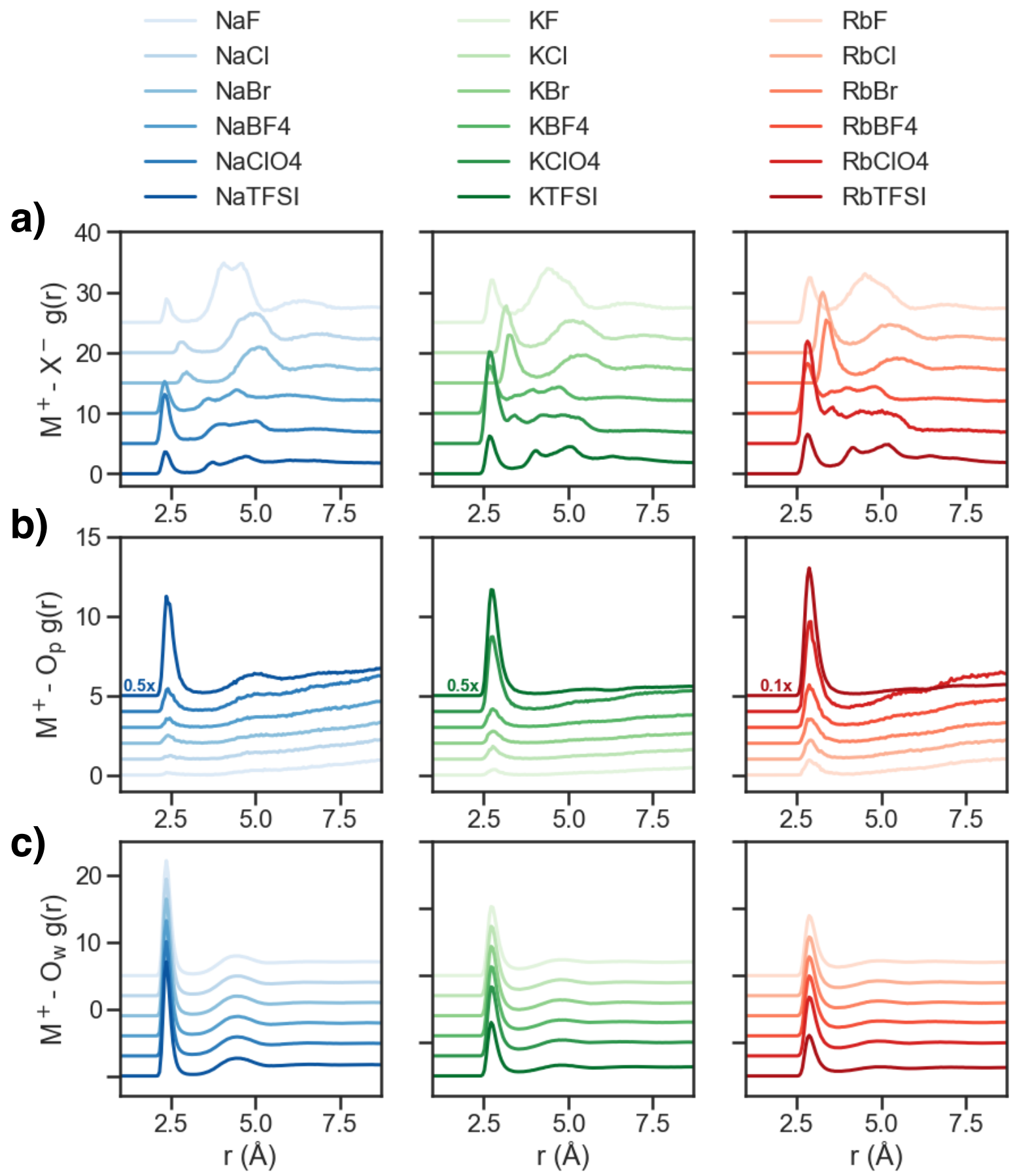

Figure 2. Radial distribution function $\mathrm{g}(\mathrm{r})$ between $\mathrm{M}^{+}=\mathrm{Na}^{+}, \mathrm{K}^{+}$and $\mathrm{Rb}^{+}$and: a) their negative counterions $\left(\mathrm{X}^{-}=\mathrm{F}^{-}\right.$, $\left.\mathrm{Cl}^{-}, \mathrm{Br}^{-}, \mathrm{BF}_{4}^{-}, \mathrm{ClO}_{4}^{-}, \mathrm{TFSI}^{-}\right)$, b) EG side chains oxygens $\mathrm{O}_{\mathrm{p}}$ and c) water oxygens $\mathrm{O}_{\mathrm{w}}$. Curves have been vertically shifted for clarity. 
We then took a closer look at the cation-polymer interactions, quantifying the fraction of cations coordinated to at least one oxygen from the $\mathrm{p}(\mathrm{g} 2 \mathrm{~T}-\mathrm{TT})$ side chains for any given MD snapshot (Figure 3a). The probability jumps by over one order of magnitude when swapping a halogen salt with a TFSI- salt. This effect is also amplified by choosing a softer cation: as seen in Figure 2, $\mathrm{Na}^{+}$ is significantly less likely to shed water molecules from its coordination shell and allow the glycolated side chains to replace them; $\mathrm{K}^{+}$and $\mathrm{Rb}^{+}$instead show a higher propensity to swap their loosely bound water shell for $\mathrm{p}(\mathrm{g} 2 \mathrm{~T}-\mathrm{TT})$.

In order to distinguish between simple coordination and chelation, we analyzed how many side chain oxygen atoms were found within a $4 \AA$ radius from each metal ion. Figure 3 b shows how softer counterions not only increase the overall probability of polymer coordination, but also promote chelation - defined as coordination by at least two EG oxygens. In the case of $\mathrm{Na}^{+}$salts, chelation is almost absent when harder anions are present; in NaTFSI it is the prevalent kind of interaction. $\mathrm{K}^{+}$and $\mathrm{Rb}^{+}$salts show chelation even in the presence of fluoride, but polymer side chains can completely saturate their coordination sphere only if TFSI- is used as counterion. To conclude, diffuse and larger anions, having less solvating water, can more easily penetrate the first coordination shell of cations; this in turn makes cations available to chelating EG oxygens. 
b)
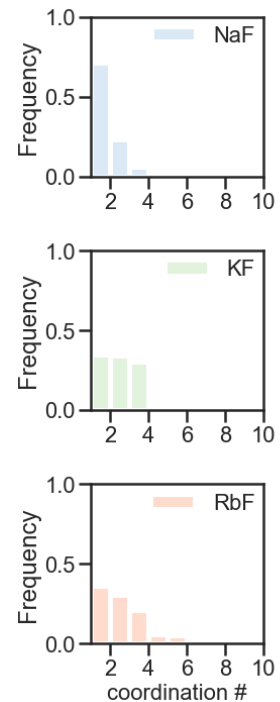

a)
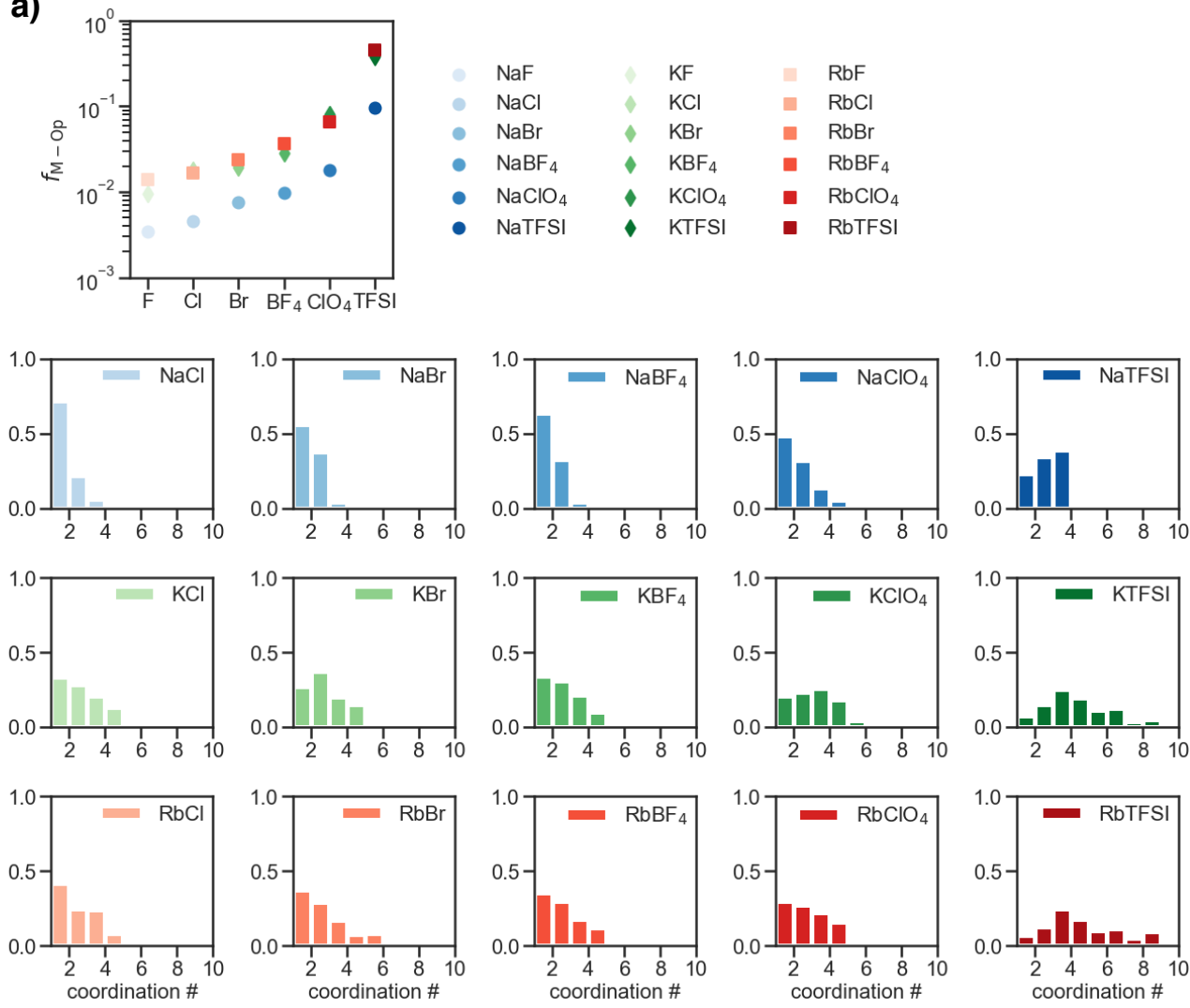

Figure 3. a) Fraction of alkali metal ions coordinated to $\mathrm{p}(\mathrm{g} 2 \mathrm{~T}-\mathrm{TT})$, defined as the number of ions having at least one EG oxygen within $4 \AA$, normalized by the total number of cations in the simulation box over 100 simulation snapshots. b) Normalized frequency of coordination numbers for alkali ions, defined as the number of oxygen atoms from $\mathrm{p}(\mathrm{g} 2 \mathrm{~T}-\mathrm{TT})$ within a $4 \AA$ radius over 100 simulation snapshots.

\section{Water-polymer hydrogen bonds and p(g2T-TT) surface area}

Looking at the hydrogen bonds between the $\mathrm{p}(\mathrm{g} 2 \mathrm{~T}-\mathrm{TT})$ side chains and the solvent can provide us with indirect information on the availability of the side chains at the interface. As expected, the average number of hydrogen bonds is roughly independent of the electrolyte, since all simulations have in common the initial polymer conformation (see Figure 4a). However, KTFSI and RbTFSI strongly deviate from the general picture: EG side chains are consistently less available to make hydrogen bonds, which is in line with the stronger chelation tendency discussed previously.

Figure 4b reports the p(g2T-TT) solvent accessible surface area (SASA) for all studied electrolyte interfaces. Overall, the effect of the electrolyte on the surface area of the polymer appears generally 
modest, except when TFSI- is present. In presence of RbTFSI and KTFSI salts, p(g2T-TT) shows markedly higher $<$ SASA $>$ values. This observation, together with the sharp decrease in hydrogen bonds discussed earlier, confirms the increased interaction between polymer and electrolyte. We then examined the fraction of surface area corresponding to the EG side chain oxygen atoms (Figure 4c): the stacked histograms show the separate contributions from oxygens situated at different distances from the polymer backbone. All TFSI' salts show an increase in the exposed oxygen surface area, which concerns all but the oxygen closest to the backbone. These results are in line with the higher hydrophobicity of $\mathrm{TFSI}^{-}$, as well as with previous observations concerning the behavior of this and other hydrophobic, larger ions. ${ }^{27,28}$ Interestingly, when $\mathrm{p}(\mathrm{g} 2 \mathrm{~T}-\mathrm{TT})$ is interfaced with NaTFSI salts, it shows only a modest decrease in the number of hydrogen bonds with water with respect to other $\mathrm{Na}$ salts, as well as a slightly lower side chain $<\mathrm{SASA}_{\mathrm{O}}>$ when compared to KTFSI or RbTFSI. This is related to the previously discussed lower coordination tendency of $\mathrm{Na}$ to $\mathrm{p}(\mathrm{g} 2 \mathrm{~T}-\mathrm{TT})$ due to a strongly bound hydration shell, showing that harder cations can effectively modulate the anion-polymer interface.

In summary, we have observed how the size and hardness of both cations and anions play a significant role in modulating the interactions between alkali metals and $\mathrm{p}(\mathrm{g} 2 \mathrm{~T}-\mathrm{TT})$. In this context, the strongest effect is seen with TFSI- salts, in which metal-polymer chelation becomes strongly favored. This is not unexpected, given the size and charge density of this polyatomic anion. In the next section, we investigate how different anions directly affect the polymer interface by looking at the density and electrostatic potential profiles. 
a)

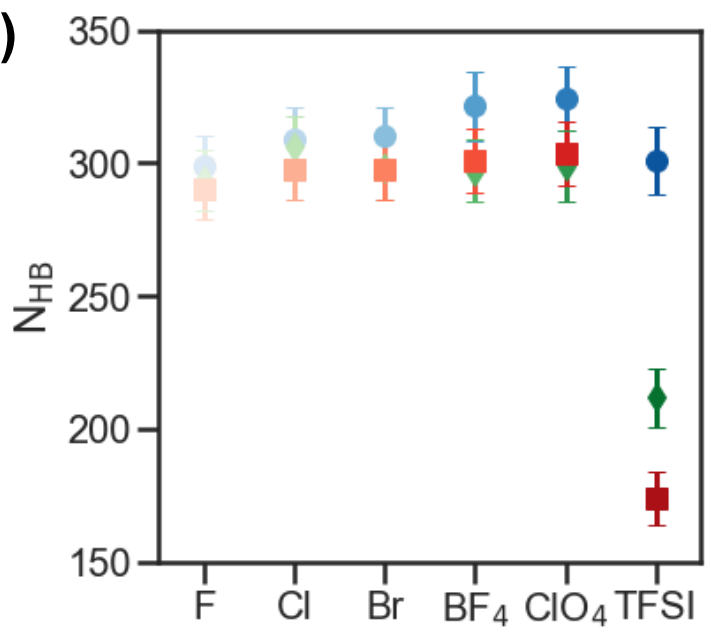

b)

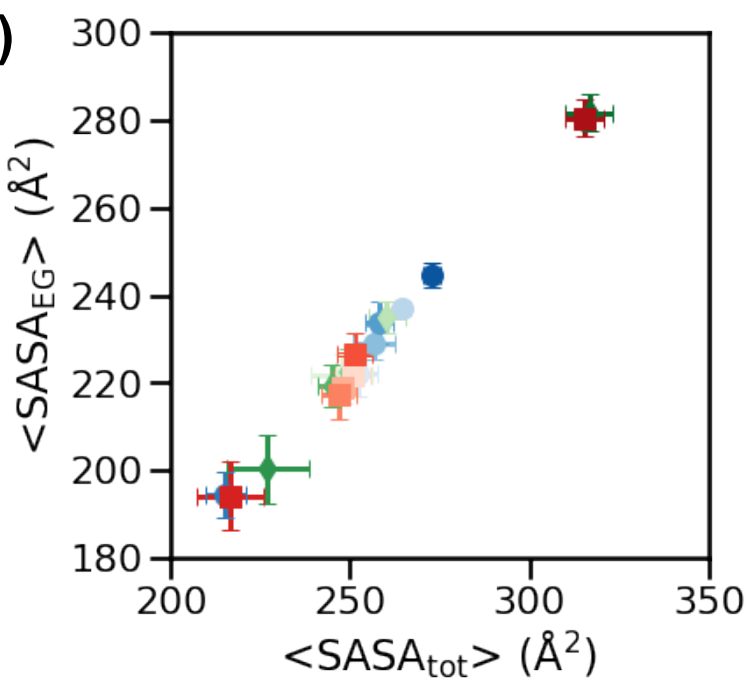

c)

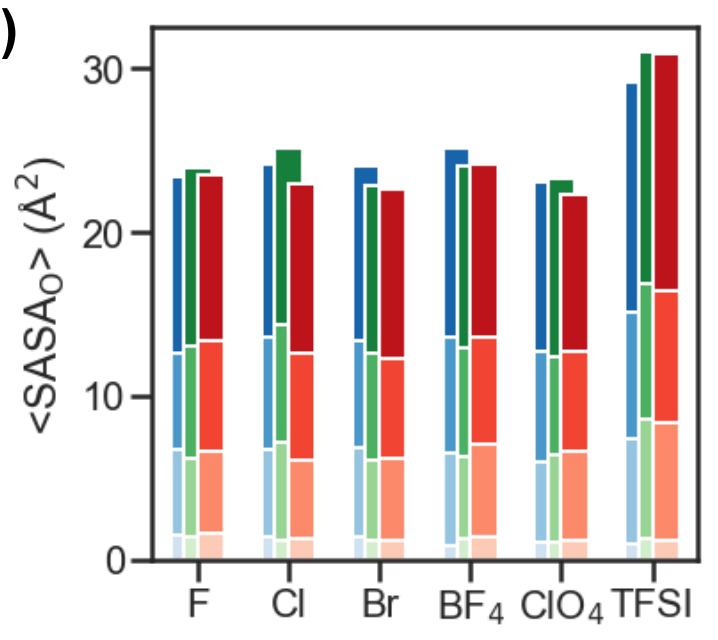

$-\mathrm{NaF}-\mathrm{KF}-\mathrm{RbF}$

$+\mathrm{NaCl}+\mathrm{KCl}+\mathrm{RbCl}$

$+\mathrm{NaBr}+\mathrm{KBr}+\mathrm{KbBr}$

$+\mathrm{NaBF}_{4}+\mathrm{KBF}_{4}+\mathrm{RbBF}_{4}$

$+\mathrm{NaClO}_{4}+\mathrm{KClO}_{4}+\mathrm{RbClO}_{4}$

NaTFSI + KTFSI RbTFSI

Figure 4. a) Average number of hydrogen bonds between water and $\mathrm{p}(\mathrm{g} 2 \mathrm{~T}-\mathrm{TT})$. b) Average solvent accessible surface area, $<$ SASA $>$. Blue dots: $\mathrm{Na}^{+}$salts; green diamonds: $\mathrm{K}^{+}$salts; red squares: $\mathrm{Rb}^{+}$salts. c) Breakdown of the contribution to the $\mathrm{p}(\mathrm{g} 2 \mathrm{~T}-\mathrm{TT}) \mathrm{SASA}$ from side chain oxygen atoms, $<\mathrm{SASA}_{\mathrm{O}}>$, where $\mathrm{O}_{1}$ is the oxygen closest to the backbone and $\mathrm{O}_{4}$ is the farthest.

\section{Density and electrostatic potential profiles at the water-p(g2T-TT) interface}

To probe the existence of specific anion-polymer interactions, we calculated the RDF between anions and either EG side chain oxygens or backbone sulfur atoms (Figure S1). Unlike halogens where no specific interactions or close contacts are present, larger anions like $\mathrm{BF}_{4}^{-}, \mathrm{ClO}_{4}{ }^{-}$and TFSI$^{-}$show a higher affinity with the polymer. The EG side chain-anion RDF increases with 
increasing cation coordination, with peaks around 5 A. For KTFSI and RbTFSI this RDF peak is almost unity; this result is consistent with the highest $<\mathrm{SASA}_{O}>$ calculated for these systems. (Figure S1a). In the case of $\mathrm{ClO}_{4}^{-}$and $\mathrm{TFSI}^{-}$, the two most hydrophobic ions studied, the RDFs show the presence of close contacts with the exposed polymer backbone (Figure S1b). These are accompanied by corresponding peaks in the cation-sulfur RDF (Figure S1c).

We then calculated the electrostatic potential profile across the simulation box, shown in Figure 5a and Figure S2. The drop from higher to lower potential is identifiable as the interface region between $\mathrm{p}(\mathrm{g} 2 \mathrm{~T}-\mathrm{TT})$ and water. The lower contrast between the two phases is observed both with softer cations (ie, KF and RbF vs $\mathrm{NaF}$ ) and with larger anions, particularly TFSI-. The density profiles of all electrolytes and p(g2T-TT) in Figure $\mathbf{5 b}$ reveal how TFSI- tends to concentrate at the water-p(g2T-TT) interface. This is seen also for $\mathrm{ClO}_{4}$ salts, albeit to a lower extent (see also Figure S3). Interestingly, while with $\mathrm{Rb}^{+}$and $\mathrm{K}^{+}$the concentration of TFSI- is highest at the polymer interface, the presence of $\mathrm{Na}$ increases the solubility of TFSI- in water, as seen from the higher density in the water phase. This observation also matches the lowest drop in electrostatic potential seen for NaTFSI. Overall, the different behavior of $\mathrm{TFSI}^{-}$and $\mathrm{ClO}_{4}^{-}$can be explained considering the higher hydrophobicity of these anions. A closer look at the polymer-electrolyte close contacts for KTFSI and RbTFSI is shown in Figure $\mathbf{S 4}$.

In summary, our MD simulations showed the importance of different anions in the modulation of polymer-electrolyte interactions. The use of large and softer anions not only enhances metalpolymer chelation, but in some cases results in a modification of the polymer-water interface due to the marked hydrophobic character of species like TFSI-. In the next section, we discuss the experimental validation of these results carried out via X-ray fluorescence for a series of TFSI salts, with the aim of quantifying the TFSI-polymer interactions and TFSI-enhanced metal chelation. 

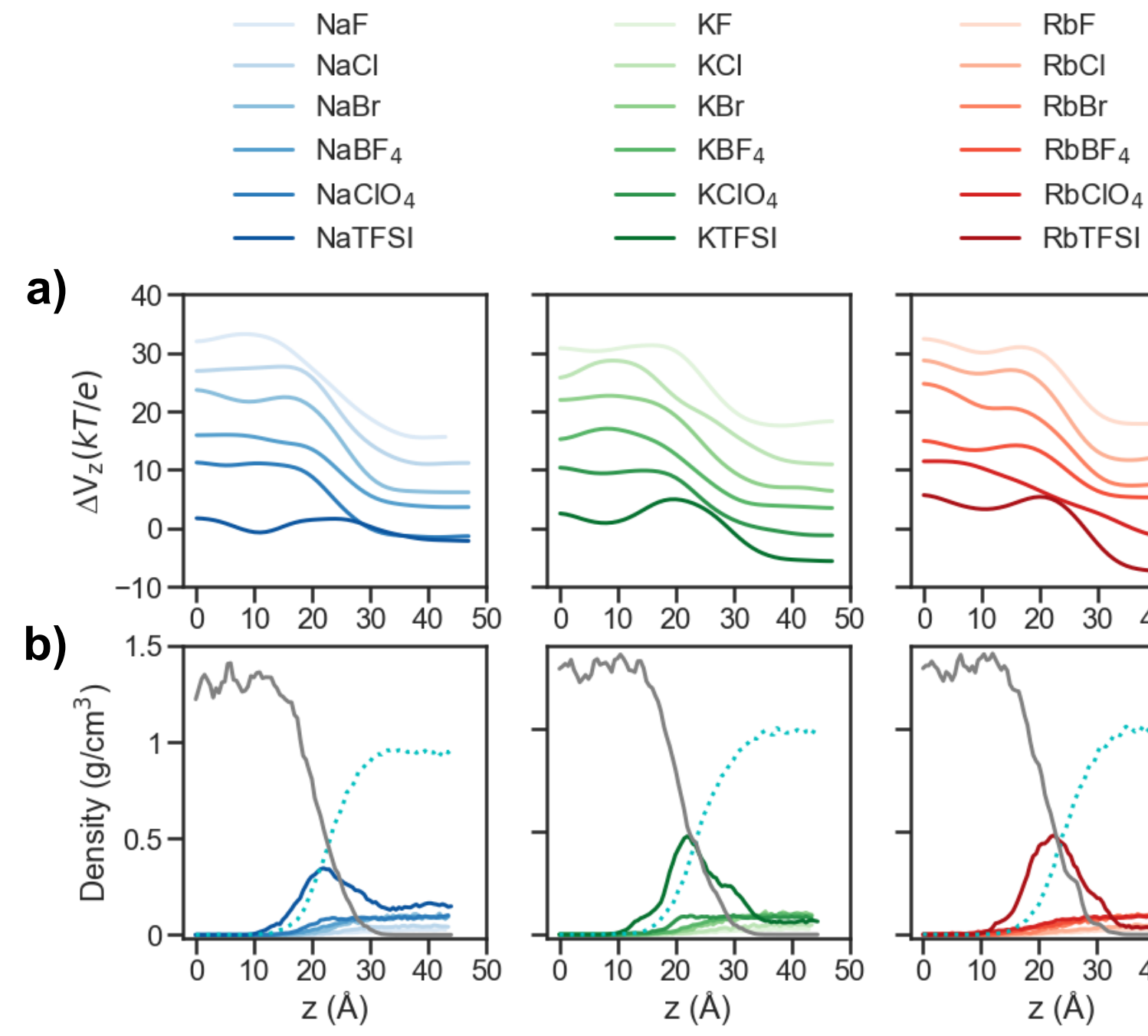

a)
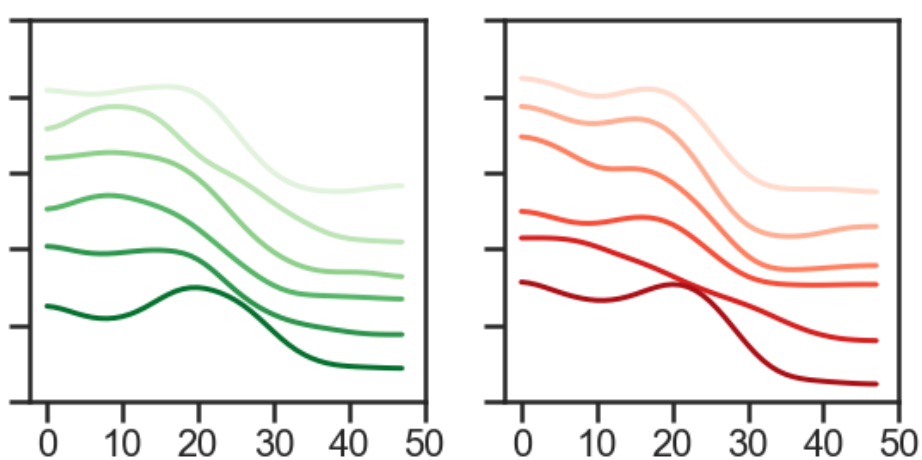

Figure 5. a) Change in electrostatic potential $\Delta V$ along the vertical axis z. The polymer phase, corresponding to the higher potential region, is on the right side. Curves are vertically shifted for clarity. b) Density profile along the $\mathrm{z}$ axis of $\mathrm{p}$ (g2T-TT) (grey line), water (cyan dots) and anions (color gradient solid lines).

\section{X-ray fluorescence study of OECTs with Rb and K salts}

Quantifying the ion-polymer interactions in electrolyte infiltrated OMIECs presents a difficult experimental task. X-ray techniques provide one route to probe ion composition and local ion environment. Therefore, ex situ X-ray fluorescence and absorption measurements were carried out on electrolyte exposed films of $\mathrm{p}(\mathrm{g} 2 \mathrm{~T}-\mathrm{TT})$. X-ray fluorescence (XRF) was carried out on neat films and films exposed to $\mathrm{K}^{+}$and $\mathrm{Rb}^{+}$based electrolytes, as shown in Figure 6 (the $\mathrm{Na}^{+} \mathrm{K}_{\alpha}$ peak energy of $1.04 \mathrm{keV}$ was outside the energy range of the experimental apparatus, thus $\mathrm{Na}$ based salts where 
excluded). The TFSI- induced metal-polymer chelation was measurable as an increase in X-ray fluorescence (XRF) as more cations were expected to be entrained in TFSI-based electrolyte exposed films of p(g2T-TT), as compared to those exposed to metal-halogen electrolytes. Films of $\mathrm{p}$ (g2T-TT) ex situ exposed to $1 \mathrm{M}$ aqueous $\mathrm{KCl}, \mathrm{KBr}, \mathrm{RbCl}$, and $\mathrm{RbBr}$ showed no evidence of retained cations (i.e. absence of resolvable $\mathrm{K}$ or $\mathrm{Rb} \mathrm{K}_{\alpha}$ peaks). Accounting for the noise floor of the $\mathrm{X}$-ray detector, in the case of $\mathrm{Rb}^{+}$this would imply a cation concentration of at most 0.005 cations per heterocyclic sulfur. Conversely, films of $\mathrm{p}(\mathrm{g} 2 \mathrm{~T}-\mathrm{TT})$ ex situ exposed to $1 \mathrm{M}$ aqueous KTFSI and RbTFSI showed a clear $\mathrm{K}$ and $\mathrm{Rb} \mathrm{K}_{\alpha}$ peaks, respectively, which matches the predicted anion-enabled chelation.

a)

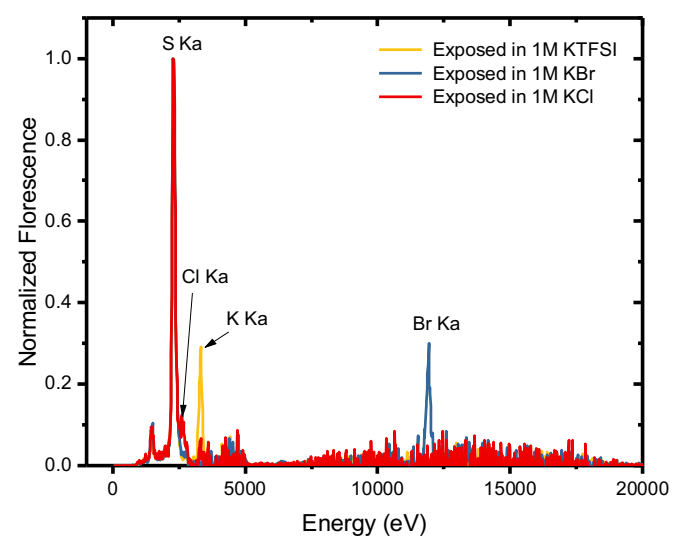

b)

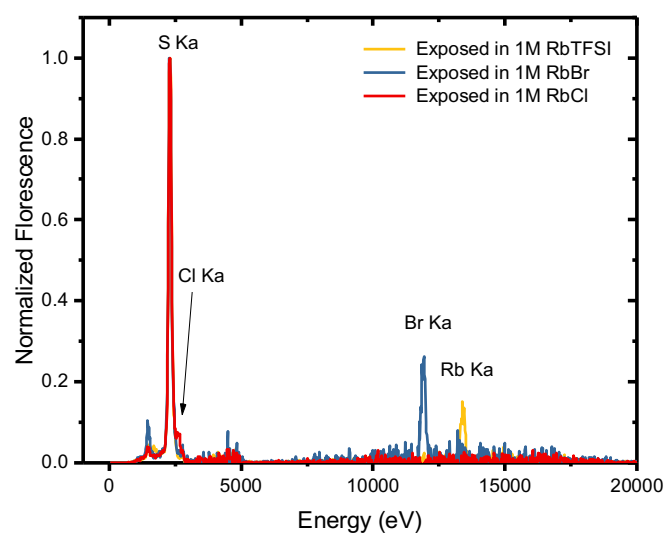

Figure 6. XRF spectra of p(g2T-TT) films ex situ exposed to 1M aqueous solutions of a) $\mathrm{KTFSI}, \mathrm{KBr}$ and $\mathrm{KCl}$, and b) $\mathrm{RbTFSI}, \mathrm{RbBr}$ and $\mathrm{RbCl}$, each normalized to $\mathrm{S}_{\alpha}$ fluorescence.

While the simulations do not account for charge on the polymer backbone, it is expected that $\mathrm{p}(\mathrm{g} 2 \mathrm{~T}-\mathrm{TT})$ processed in ambient be oxidized to some degree. This is manifest in the XRF spectra of films exposed to $\mathrm{KCl}, \mathrm{KBr}, \mathrm{RbCl}$, and $\mathrm{RbBr}$ which, while showing no measurable cation fluorescence, displayed fluorescence from anions $\left(\mathrm{Cl}^{-}\right.$or $\left.\mathrm{Br}^{-}\right)$presumably balancing positive charge on the partially oxidized $\mathrm{p}(\mathrm{g} 2 \mathrm{~T}-\mathrm{TT})$. Similarly, the $\mathrm{S} \mathrm{K}_{\alpha}$ peak increased following exposure to KTFSI and RbTFSI. Due to the large separation in energies of $\mathrm{S}, \mathrm{Br}$, and $\mathrm{Rb} \mathrm{K}_{\alpha}$ peaks, these presented an ideal case to convert the qualitative spectral change to quantitative anion and cation compositional changes. Subtracting the XRF spectra collected before and after electrolyte exposure revealed a consistent 15 to $17 \%$ increase in S fluorescence, representing an entrained 
anion concentration of one TFSI- per $\sim 12.5$ heterocyclic sulfurs or one TFSI$^{-}$per $\sim 3$ repeat units. Known $\mathrm{S}, \mathrm{Br}$, and $\mathrm{Rb}$ composition samples were prepared and measured with XRF to produce compositional calibration curves. Samples exposed to $\mathrm{KBr}$ and $\mathrm{RbBr}$ displayed an uptake of 0.047 and $0.048 \mathrm{Br}^{-}$per heterocyclic sulfur, respectively. This translates to around one $\mathrm{Br}^{-}$every five repeats unit for both $\mathrm{KBr}$ and $\mathrm{RbBr}$ exposed films. As mentioned above, this $\mathrm{Br}^{-}$is presumed to be balancing positive charge on the partially oxidized $\mathrm{p}(\mathrm{g} 2 \mathrm{~T}-\mathrm{TT})$.

Samples exposed to RbTFSI displayed an uptake of $0.051 \mathrm{Rb}^{+}$ions per heterocyclic sulfur and 0.079 TFSI $^{-}$per heterocyclic sulfur. The excess TFSI $^{-}$is consistent with the halogen anion concentration present to counterbalance the positive charge on $\mathrm{p}(\mathrm{g} 2 \mathrm{~T}-\mathrm{TT})$ due to its partially oxidized state. The oxidation of the polymer cannot explain the preferential uptake of $\mathrm{Rb}^{+}$when the TFSI ${ }^{-}$anion is employed since both RbTFSI and RbBr show a similar anion uptake due to the partial $\mathrm{p}(\mathrm{g} 2 \mathrm{~T}-\mathrm{TT})$ oxidation. Thus, it seems highly likely that the presence of $\mathrm{Rb}^{+}$cations is due to the predicted TFSI- enabled cation-sidechain chelation. $\mathrm{KCl}, \mathrm{KBr}$, and $\mathrm{KTFSI}$ followed the same qualitative trends. The TFSI- enabled cation-sidechain chelation was observed to be reversible with no cation or anion XRF signal present after multiple soak and rinse cycles with DI water.

Extended X-ray absorption fine structure (EXAFS) was carried out on p(g2T-TT) films exposed $\mathrm{Rb}^{+}$based electrolytes. The high level of disorder, relative long length scales, and difficulties with contrast undermined the effectiveness of ex situ EXAFS. Ambient EXAFS at the $\mathrm{Rb}^{+} \mathrm{K}$ edge lacked the resolution to confirm RDFs or discern changes in the Rb environment due to anion choice.

\section{Conclusion}

In summary, we have carried out both XRF and MD simulations on a series of p(g2T-TT)electrolyte interfaces. We have analyzed the effect of different counterions on the p(g2T-TT)-water interface, and their ability to modulate coordination and chelation of metal cations. We have shown how softer polyatomic anions, particularly $\mathrm{TFSI}^{-}$and $\mathrm{ClO}_{4}^{-}$, can enhance metal coordination and chelation by EG side chains. The higher hydrophobic character of TFSI- results in a tendency to concentrate at the $\mathrm{p}(\mathrm{g} 2 \mathrm{~T}-\mathrm{TT})$ interface, increasing both the surface area of the polymer and the 
cation-side chain chelation. On the other hand, the use of smaller, harder cations such as $\mathrm{Na}^{+}$can mitigate this effect by increasing the solubility of TFSI in water. These trends are consistent with the literature on PEO-water interfaces, ${ }^{29-32}$ and they may translate in significant differences in the context of operating OECTs.

Our results can be directly compared to a working OECT in the 'off' state undergoing passive uptake. The trends observed in this work open new discussions and speculations on how the choice of the electrolyte might affect the device morphology and OECT operation, not only in the specific case of $\mathrm{p}(\mathrm{g} 2 \mathrm{~T}-\mathrm{TT})$ but for the general family of glycolated polymers. In this respect, different salts could correspond to different doping/dedoping kinetics, ${ }^{33,34}$ or even modify the microstructure of the polymer, as already seen experimentally for $\mathrm{p}(\mathrm{g} 2 \mathrm{~T}-\mathrm{TT})$. Overall, our data suggest that the choice of the electrolyte should be an important parameter when testing OECTs; this is particularly relevant for applications such as neuromorphic devices, batteries or supercapacitors that do not involve biological substrates, where strict boundaries of $\mathrm{pH}$ and biocompatibility do not apply.

Finally, we highlight the importance of theory and simulations in investigating the dynamics and structure of mixed transport materials. MD simulations, coupled with ab-initio or semiempirical methods aimed at capturing polarization effects on the polymer electronic structure, will prove a robust tool to gain a general understanding of OMIECs and develop a theory of mixed transport able to guide the rational design of polymer mixed conductors. In this context, the simulations performed in this work will be the basis of further investigations on the doping kinetics of $\mathrm{p}(\mathrm{g} 2 \mathrm{~T}$ TT), for which a rigorous theoretical model is still lacking. 


\section{Computational methods}

Force field and software details. The general amber force field (GAFF) ${ }^{35}$ for $\mathrm{p}(\mathrm{g} 2 \mathrm{~T}-\mathrm{TT})$ was reparameterized for the g2T-TT repeating unit, with RESP partial charges obtained at B3LYP/6$31 \mathrm{G}^{*}$ level. The torsional parameters for dihedrals between aromatic units were obtained from fitting B3LYP/6-31G*/MP2 potential energy surface scans. DFT and MP2 calculations were performed using GAMESS-US ${ }^{36}$ and ORCA. ${ }^{37}$ The water model SPC/E ${ }^{38}$ was chosen because of its proven reliability in high ionic strength environments. ${ }^{39}$ Force field parameters for $\mathrm{BF}_{4}^{40}$ and TFSI ${ }^{41}$ ions were taken from Agilio Padua's GitHub repository; ${ }^{42}$ those for $\mathrm{ClO}_{4}{ }^{43}$ from Bryce's Amber parameter database. ${ }^{44}$ The systems were prepared using PACKMOL ${ }^{45}$ and antechamber. ${ }^{46}$ Molecular dynamics were performed using Amber16. ${ }^{47}$ The post-production analysis was performed using in-house Python code based on the MDAnalysis library. ${ }^{48,49}$

System setup. A total of 20 polymer chains of 16 repeating units were simulated. The polymer film was built by merging two pre-annealed polymer bundles containing 10 chains each and annealing the system under 3D periodic boundary conditions at $500 \mathrm{~K}$ until a stationary density was achieved. A layer of SPC/E water was then added to simulate a planar interface and the system was re-equilibrated at $298.15 \mathrm{~K}$ for $100 \mathrm{~ns}$. Finally, different alkali salts (cations: $\mathrm{Na}^{+}, \mathrm{K}^{+}, \mathrm{Rb}^{+}$;

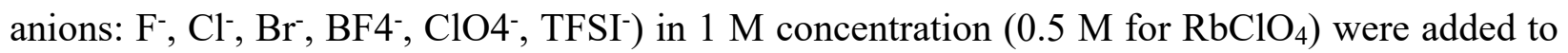
the pre-equilibrated interfaces and the resulting system was simulated for an additional $600 \mathrm{~ns}$. The 20 polymer chains consisting of 16 repeating units each are oriented with the backbone parallel to the $\mathrm{y}$ axis; the water-polymer interface is along the $\mathrm{z}$ axis.

\section{Experimental Materials and Methods}

Potassium chloride ( $>99.0 \%)$, potassium bromide $(>99.0 \%)$, potassium carbonate $(99.8 \%)$, and rubidium carbonate $(99.8 \%)$ were purchased from Sigma Aldrich; bis(trifluoromethane)sulfonimide (>99.0\%) was purchased from TCI America; rubidum chloride (99.8\%) and rubidium bromide (99.8\%) were purchased from Alfa Aesar. Potassium bis(trifluoromethane)sulfonimide ad rubidium bis(trifluoromethane)sulfonimide were synthesized following a modified previously published method. ${ }^{50}$ Poly(2-(3,3'-bis(2-(2-(2- 
methoxyethoxy)ethoxy)ethoxy)-[2,2'-bithiophen]-5-yl)thieno[3,2-b]thiophene) was synthesized following previously published methods. ${ }^{17}$

Thin films samples of $\mathrm{p}(2 \mathrm{~T}-\mathrm{TT})$ were drop cast in a saturated chloroform environment on $25 \mu \mathrm{m}$ polyimide films from $5 \mathrm{mg} / \mathrm{ml}$ solutions in chloroform. Prior to drop casting, polyimide films were sonicated in acetone and isopropyl alcohol followed by a $10 \mathrm{~min} \mathrm{UV}$-ozone exposure. Prior to electrolyte exposure, films were soaked and rinsed with deionized (DI) water several times to remove any metal-halide impurities. Ex situ electrolyte exposure was performed by immersing the thin film samples in $1 \mathrm{M}$ aqueous salt solutions for 30 minutes. Following exposure, thin film samples were quickly rinsed with DI water to displace liquid electrolyte form the surface and blown dry with nitrogen flow.

X-ray fluorescence spectra was collected with a Xenemetrix ED-XRF-measure unit with a Rhodium white light X-ray source. The energy range was set at $20 \mathrm{keV}$. In order to improve the signal-to-background ratio for light elements including $\mathrm{S}, \mathrm{Cl}$ and $\mathrm{K}$, a Titanium filter was placed between the X-ray source and sample to remove the Rh L lines. The measurement was carried out under vacuum with 300-second exposures. For the data analysis, peaks were simulated by single gaussian peaks to quantify their positions and areas. A calibration curve was constructed by measuring a series of known composition mixtures of thiourea and Rubidium salts spin-coated on $25 \mu \mathrm{m}$ polyimide films. EXAFS was carried out on the bending magnet beamline in Sector 5 (DND-CAT) of the Advanced Photon Source at Argonne National Laboratory.

\section{ASSOCIATED CONTENT}

\section{Supporting Information}

Additional analysis of MD simulations, individual XRF spectra, synthetic details and NMR spectra are available as a pdf file.

\section{AUTHOR INFORMATION}

\section{Corresponding Author}


micaela.matta@liverpool.ac.uk

\section{Author Contributions}

M.M. performed the simulations, analyzed the results and drafted the manuscript. M.M., R.W., B.D.P., and J.R conceived the experiments. R.W. and B.D.P. carried out the experiments and analyzed the results. A.J.P. and R.S. synthesized and characterized the TFSI salts and conjugated polymer, respectively. All authors contributed to the writing of the manuscript.

\section{Acknowledgements}

M.M. is grateful to Prof. Alessandro Troisi for useful comments during the preparation of this manuscript. R.W. and B.D.P. are grateful to Dr. Christos D. Malliakas for helpful discussion.

\section{Funding Sources}

MM and GCS were supported by NSF grant CMMI-1848613. RW BDP, and JR gratefully acknowledge support from the National Science Foundation Grant No. NSF DMR-1751308. This work used the Extreme Science and Engineering Discovery Environment (XSEDE) BRIDGES at the Pittsburgh Supercomputing Center (PSC) through allocation CHE190029. This work made use of the IMSERC at Northwestern University, which has received support from the Soft and Hybrid Nanotechnology Experimental (SHyNE) Resource (NSF ECCS-1542205), the State of Illinois, and the International Institute for Nanotechnology (IIN). Portions of this work were performed at the DuPont-Northwestern-Dow Collaborative Access Team (DND-CAT) located at Sector 5 of the Advanced Photon Source (APS). DND-CAT is supported by Northwestern University, The Dow Chemical Company, and DuPont de Nemours, Inc. This research used resources of the Advanced Photon Source, a U.S. Department of Energy (DOE) Office of Science User Facility operated for the DOE Office of Science by Argonne National Laboratory under Contract No. DE-AC02$06 \mathrm{CH} 11357$. 


\section{References}

(1) Rivnay, J.; Inal, S.; Salleo, A.; Owens, R. M.; Berggren, M.; Malliaras, G. G. Organic Electrochemical Transistors. Nat. Rev. Mater. 2018, 3 (2), 17086.

https://doi.org/10.1038/natrevmats.2017.86.

(2) Inal, S.; Rivnay, J.; Suiu, A. O.; Malliaras, G. G.; McCulloch, I. Conjugated Polymers in Bioelectronics. Acc. Chem. Res. 2018, 51 (6).

https://doi.org/10.1021/acs.accounts.7b00624.

(3) Someya, T.; Bao, Z.; Malliaras, G. G. The Rise of Plastic Bioelectronics. Nature 2016, 540 (7633), 379-385. https://doi.org/10.1038/nature21004.

(4) Picca, R. A.; Manoli, K.; Macchia, E.; Sarcina, L.; Di Franco, C.; Cioffi, N.; Blasi, D.; Österbacka, R.; Torricelli, F.; Scamarcio, G.; et al. Ultimately Sensitive Organic Bioelectronic Transistor Sensors by Materials and Device Structures' Design. Adv. Funct. Mater. 2019. https://doi.org/10.1002/adfm.201904513.

(5) Scheiblin, G.; Coppard, R.; Owens, R. M.; Mailley, P.; Malliaras, G. G. Referenceless PH Sensor Using Organic Electrochemical Transistors. Adv. Mater. Technol. 2017, 2 (2), 1600141. https://doi.org/10.1002/admt.201600141.

(6) Mitraka, E.; Gryszel, M.; Vagin, M.; Jafari, M. J.; Singh, A.; Warczak, M.; Mitrakas, M.; Berggren, M.; Ederth, T.; Zozoulenko, I.; et al. Electrocatalytic Production of Hydrogen Peroxide with Poly(3,4-Ethylenedioxythiophene) Electrodes. Adv. Sustain. Syst. 2019. https://doi.org/10.1002/adsu.201800110.

(7) Moia, D.; Giovannitti, A.; Szumska, A. A.; Maria, I. P.; Rezasoltani, E.; Sachs, M.; Schnurr, M.; Barnes, P. R. F.; McCulloch, I.; Nelson, J. Design and Evaluation of Conjugated Polymers with Polar Side Chains as Electrode Materials for Electrochemical Energy Storage in Aqueous Electrolytes. Energy Environ. Sci. 2019, 12 (4), 1349-1357. https://doi.org/10.1039/C8EE03518K.

(8) Van De Burgt, Y.; Melianas, A.; Keene, S. T.; Malliaras, G.; Salleo, A. Organic Electronics for Neuromorphic Computing. Nat. Electron. 2018, 1 (7), 386-397. 
https://doi.org/10.1038/s41928-018-0103-3.

(9) Ling, H.; Koutsouras, D. A.; Kazemzadeh, S.; van de Burgt, Y.; Yan, F.; Gkoupidenis, P. Electrolyte-Gated Transistors for Synaptic Electronics, Neuromorphic Computing, and Adaptable Biointerfacing. Appl. Phys. Rev. 2020, 7 (1), 011307.

https://doi.org/10.1063/1.5122249.

(10) Paulsen, B. D.; Tybrandt, K.; Stavrinidou, E.; Rivnay, J. Organic Mixed Ionic-Electronic Conductors. Nat. Mater. 2020, 19 (1), 13-26. https://doi.org/10.1038/s41563-019-0435-z.

(11) Fornari, R. P.; Blom, P. W. M.; Troisi, A. How Many Parameters Actually Affect the Mobility of Conjugated Polymers? Phys. Rev. Lett. 2017, 118 (8), 086601. https://doi.org/10.1103/PhysRevLett.118.086601.

(12) Noriega, R.; Salleo, A.; Spakowitz, A. J. Chain Conformations Dictate Multiscale Charge Transport Phenomena in Disordered Semiconducting Polymers. Proc. Natl. Acad. Sci. U. S. A. 2013, 110 (41), 16315-16320. https://doi.org/10.1073/pnas.1307158110.

(13) Fratini, S.; Nikolka, M.; Salleo, A.; Schweicher, G.; Sirringhaus, H. Charge Transport in High-Mobility Conjugated Polymers and Molecular Semiconductors. Nat. Mater. 2020. https://doi.org/10.1038/s41563-020-0647-2.

(14) Dijk, G.; Rutz, A. L.; Malliaras, G. G. Stability of PEDOT:PSS-Coated Gold Electrodes in Cell Culture Conditions. Adv. Mater. Technol. 2020.

https://doi.org/10.1002/admt.201900662.

(15) Zeglio, E.; Rutz, A. L.; Winkler, T. E.; Malliaras, G. G.; Herland, A. Conjugated Polymers for Assessing and Controlling Biological Functions. Advanced Materials. 2019. https://doi.org/10.1002/adma.201806712.

(16) Inal, S.; Malliaras, G. G.; Rivnay, J. Benchmarking Organic Mixed Conductors for Transistors. Nat. Commun. 2017, 8 (1), 1-6. https://doi.org/10.1038/s41467-017-01812-w.

(17) Giovannitti, A.; Sbircea, D.-T.; Inal, S.; Nielsen, C. B.; Bandiello, E.; Hanifi, D. A.; Sessolo, M.; Malliaras, G. G.; McCulloch, I.; Rivnay, J. Controlling the Mode of 
Operation of Organic Transistors through Side-Chain Engineering. Proc. Natl. Acad. Sci. 2016, 113 (43), 12017-12022. https://doi.org/10.1073/pnas.1608780113.

(18) Venkatraman, V.; Friedlein, J. T.; Giovannitti, A.; Maria, I. P.; McCulloch, I.; McLeod, R. R.; Rivnay, J. Subthreshold Operation of Organic Electrochemical Transistors for Biosignal Amplification. Adv. Sci. 2018, 5 (8). https://doi.org/10.1002/advs.201800453.

(19) Cendra, C.; Giovannitti, A.; Savva, A.; Venkatraman, V.; McCulloch, I.; Salleo, A.; Inal, S.; Rivnay, J. Role of the Anion on the Transport and Structure of Organic Mixed Conductors. Adv. Funct. Mater. 2019, 29 (5), 1807034.

https://doi.org/10.1002/adfm.201807034.

(20) Savva, A.; Cendra, C.; Giugni, A.; Torre, B.; Surgailis, J.; Ohayon, D.; Giovannitti, A.; McCulloch, I.; Di Fabrizio, E.; Salleo, A.; et al. Influence of Water on the Performance of Organic Electrochemical Transistors. Chem. Mater. 2019, 31 (3), 927-937. https://doi.org/10.1021/acs.chemmater.8b04335.

(21) Inal, S.; Rivnay, J.; Hofmann, A. I.; Uguz, I.; Mumtaz, M.; Katsigiannopoulos, D.; Brochon, C.; Cloutet, E.; Hadziioannou, G.; Malliaras, G. G. Organic Electrochemical Transistors Based on PEDOT with Different Anionic Polyelectrolyte Dopants. J. Polym. Sci. Part B Polym. Phys. 2016, 54 (2), 147-151. https://doi.org/10.1002/polb.23938.

(22) Rivnay, J.; Inal, S.; Collins, B. A.; Sessolo, M.; Stavrinidou, E.; Strakosas, X.; Tassone, C.; Delongchamp, D. M.; Malliaras, G. G. Structural Control of Mixed Ionic and Electronic Transport in Conducting Polymers. Nat. Commun. 2016, 7, 11287. https://doi.org/10.1038/ncomms11287.

(23) Rivnay, J.; Leleux, P.; Ferro, M.; Sessolo, M.; Williamson, A.; Koutsouras, D. A.; Khodagholy, D.; Ramuz, M.; Strakosas, X.; Owens, R. M.; et al. High-Performance Transistors for Bioelectronics through Tuning of Channel Thickness. Sci. Adv. 2015, 1 (4), e1400251-e1400251. https://doi.org/10.1126/sciadv.1400251.

(24) Inal, S.; Malliaras, G. G.; Rivnay, J. Optical Study of Electrochromic Moving Fronts for the Investigation of Ion Transport in Conducting Polymers. J. Mater. Chem. C 2016, 4 
(18), 3942-3947. https://doi.org/10.1039/c5tc04354a.

(25) Mazzini, V.; Craig, V. S. J. What Is the Fundamental Ion-Specific Series for Anions and Cations? Ion Specificity in Standard Partial Molar Volumes of Electrolytes and Electrostriction in Water and Non-Aqueous Solvents. Chem. Sci. 2017, 8 (10), 70527065. https://doi.org/10.1039/C7SC02691A.

(26) Kim, S.; Kim, H.; Choi, J. H.; Cho, M. Ion Aggregation in High Salt Solutions: Ion Network versus Ion Cluster. J. Chem. Phys. 2014. https://doi.org/10.1063/1.4896227.

(27) Li, X.; Schatz, G. C.; Nesbitt, D. J. Anion Effects in the Scattering of CO 2 from the Room-Temperature Ionic Liquids [Bmim][BF4] and [Bmim][Tf2N]: Insights from Quantum Mechanics/Molecular Mechanics Trajectories. J. Phys. Chem. B 2012, 116 (11), 3587-3602. https://doi.org/10.1021/jp2123357.

(28) Sivan, U. The Inevitable Accumulation of Large Ions and Neutral Molecules near Hydrophobic Surfaces and Small Ions near Hydrophilic Ones. Curr. Opin. Colloid Interface Sci. 2016, 22, 1-7. https://doi.org/10.1016/J.COCIS.2016.02.004.

(29) Su, Y. L.; Liu, H. Z.; Wang, J.; Chen, J. Y. Study of Salt Effects on the Micellization of PEO-PPO-PEO Block Copolymer in Aqueous Solution by FTIR Spectroscopy. Langmuir 2002. https://doi.org/10.1021/la0106687.

(30) Deyerle, B. A.; Zhang, Y. Effects of Hofmeister Anions on the Aggregation Behavior of PEO-PPO-PEO Triblock Copolymers. Langmuir 2011. https://doi.org/10.1021/la201463g.

(31) Zajforoushan Moghaddam, S.; Thormann, E. Hofmeister Effect of Salt Mixtures on Thermo-Responsive Poly(Propylene Oxide). Phys. Chem. Chem. Phys. 2015. https://doi.org/10.1039/c4cp05677a.

(32) Thormann, E. On Understanding of the Hofmeister Effect: How Addition of Salt Alters the Stability of Temperature Responsive Polymers in Aqueous Solutions. RSC Adv. 2012. https://doi.org/10.1039/c2ra20164j.

(33) Ma, Q.; Zhang, H.; Zhou, C.; Zheng, L.; Cheng, P.; Nie, J.; Feng, W.; Hu, Y. S.; Li, H.; 
Huang, X.; et al. Single Lithium-Ion Conducting Polymer Electrolytes Based on a SuperDelocalized Polyanion. Angew. Chemie - Int. Ed. 2016.

https://doi.org/10.1002/anie.201509299.

(34) Schauser, N. S.; Seshadri, R.; Segalman, R. A. Multivalent Ion Conduction in Solid Polymer Systems. Mol. Syst. Des. Eng. 2019, 4 (2), 263-279.

https://doi.org/10.1039/c8me00096d.

(35) Wang, J.; Wolf, R. M.; Caldwell, J. W.; Kollman, P. A.; Case, D. A. Development and Testing of a General Amber Force Field. J. Comput. Chem. 2004, 25, 1157-1174. https://doi.org/10.1002/jcc.20035.

(36) Schmidt, M. W.; Baldridge, K. K.; Boatz, J. A.; Elbert, S. T.; Gordon, M. S.; Jensen, J. H.; Koseki, S.; Matsunaga, N.; Nguyen, K. A.; Su, S.; et al. GAMESS. J. Comput. Chem. 2016. https://doi.org/10.1002/jcc.540141112.

(37) Neese, F. Software Update: The ORCA Program System, Version 4.0. Wiley Interdiscip. Rev. Comput. Mol. Sci. 2018. https://doi.org/10.1002/wcms.1327.

(38) Berendsen, H. J. C.; Grigera, J. R.; Straatsma, T. P. The Missing Term in Effective Pair Potentials. J. Phys. Chem. 1987. https://doi.org/10.1021/j100308a038.

(39) Joung, S.; Cheatham, T. E. Molecular Dynamics Simulations of the Dynamic and Energetic Properties of Alkali and Halide Ions Using Water-Model-Specific Ion Parameters. J. Phys. Chem. B 2009. https://doi.org/10.1021/jp902584c.

(40) Canongia Lopes, J. N.; Deschamps, J.; Pádua, A. A. H. Modeling Ionic Liquids Using a Systematic All-Atom Force Field. J. Phys. Chem. B 2004. https://doi.org/10.1021/jp0476996.

(41) Canongia Lopes, J. N.; Pádua, A. A. H.; Shimizu, K. Molecular Force Field for Ionic Liquids IV: Trialkylimidazolium and Alkoxycarbonyl-Imidazolium Cations; Alkylsulfonate and Alkylsulfate Anions. J. Phys. Chem. B 2008. https://doi.org/10.1021/jp800281e. 
(42) Padua, A. Force field for ionic liquids https://github.com/agiliopadua/ilff. https://doi.org/10.5281/zenodo.593705.

(43) Baaden, M.; Berny, F.; Madic, C.; Wipff, G. M3+ Lanthanide Cation Solvation by Acetonitrile: The Role of Cation Size, Counterions, and Polarization Effects Investigated by Molecular Dynamics and Quantum Mechanical Simulations. J. Phys. Chem. A 2000. https://doi.org/10.1021/jp001352v.

(44) Bryce, R. AMBER parameter database http://research.bmh.manchester.ac.uk/bryce/amber/.

(45) Martinez, L.; Andrade, R.; Birgin, E. G.; Martínez, J. M. PACKMOL: A Package for Building Initial Configurations for Molecular Dynamics Simulations. J. Comput. Chem. 2009. https://doi.org/10.1002/jcc.21224.

(46) Wang, J.; Wang, W.; Kollman, P. a; Case, D. a. Antechamber, An Accessory Software Package For Molecular Mechanical Calculations. J. Am. Chem. Soc 2001.

(47) Case, D. A.; Betz, R. M.; Cerutti, D. S.; T.E., C. I.; Darden, T. A.; Duke, R. E.; Giese, T. J.; Gohlke, H.; Goetz, A. W.; Homeyer, N.; et al. Amber 2016. Univ. California, San Fr. 2016.

(48) Gowers, R.; Linke, M.; Barnoud, J.; Reddy, T.; Melo, M.; Seyler, S.; Domański, J.; Dotson, D.; Buchoux, S.; Kenney, I.; et al. MDAnalysis: A Python Package for the Rapid Analysis of Molecular Dynamics Simulations. In Proceedings of the 15th Python in Science Conference; 2016. https://doi.org/10.25080/majora-629e541a-00e.

(49) Michaud-Agrawal, N.; Denning, E. J.; Woolf, T. B.; Beckstein, O. MDAnalysis: A Toolkit for the Analysis of Molecular Dynamics Simulations. J. Comput. Chem. 2011. https://doi.org/10.1002/jcc.21787.

(50) Xue, L.; Padgett, C. W.; DesMarteau, D. D.; Pennington, W. T. Synthesis and Structures of Alkali Metal Salts of Bis[(Trifluoromethyl)Sulfonyllimide. Solid State Sci. 2002. https://doi.org/10.1016/S1293-2558(02)00050-X. 


\section{Supporting Information}

\section{Ion Coordination and Chelation in a Glycolated Polymer \\ Semiconductor: Molecular Dynamics and X-Ray Fluorescence \\ Study}

Micaela Matta*1,2, Ruiheng Wu ${ }^{3}$, Bryan D. Paulsen ${ }^{3}$, Anthony J. Petty II, ${ }^{3}$ Rajendar

Sheelamanthula, ${ }^{4}$ Iain McCulloch, ${ }^{4,5}$ George C. Schatz ${ }^{1}$, Jonathan Rivnay ${ }^{3}$

${ }^{1}$ Northwestern University, Department of Chemistry, 2145 Sheridan Rd, Evanston, IL 60208, USA

${ }^{2}$ University of Liverpool, Department of Chemistry, Crown St., Liverpool L69 7ZD, United Kingdom

${ }^{3}$ Northwestern University, Department of Biomedical Engineering, 2145 Sheridan Rd, Evanston, IL 60208, USA

${ }^{4}$ King Abdullah University of Science and Technology (KAUST), KAUST Solar Center (KSC), Thuwal 23955-6900, Saudi Arabia

${ }^{5}$ Imperial College London, Department of Chemistry and Center for Plastic Electronics, London, W12 0BZ, United Kingdom

*micaela.matta@liverpool.ac.uk

University of Liverpool, Department of Chemistry, Crown st., Liverpool L69 7ZD, United Kingdom

Table of Contents

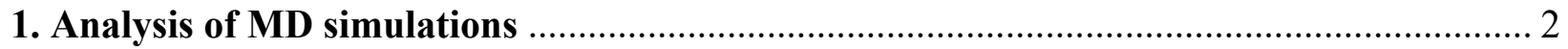

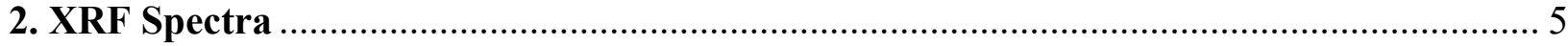

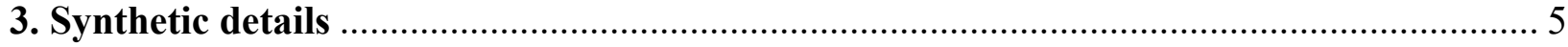

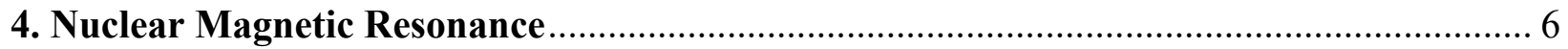




\section{Analysis of Molecular Dynamics Simulations}

a)
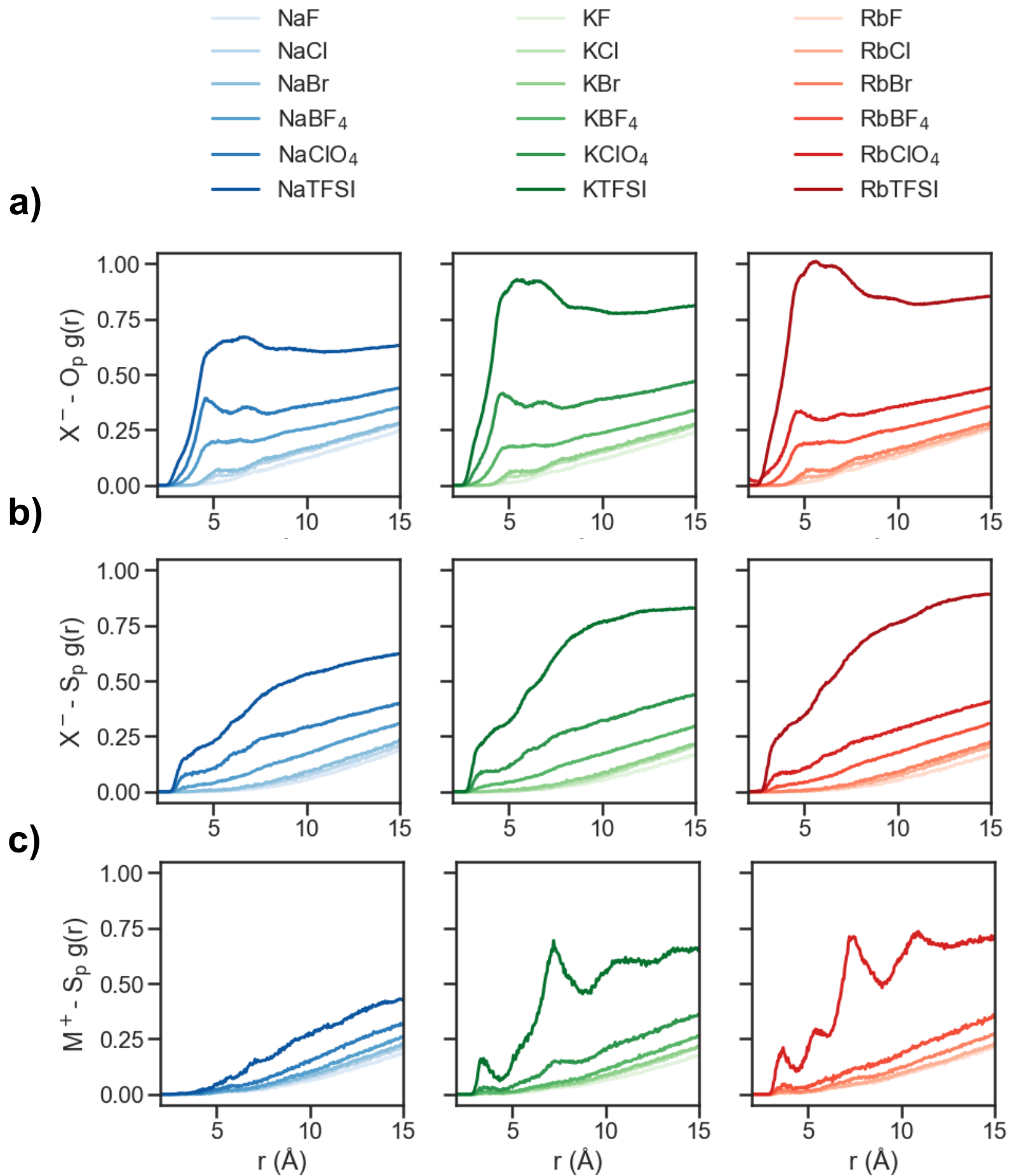

Figure S1. Radial distribution function $\mathrm{g}(\mathrm{r})$ of anions against a) the polymer backbone sulfur and b) the EG side chain oxygen. c) Radial distribution function $g(r)$ of cations against the polymer backbone sulfur. 

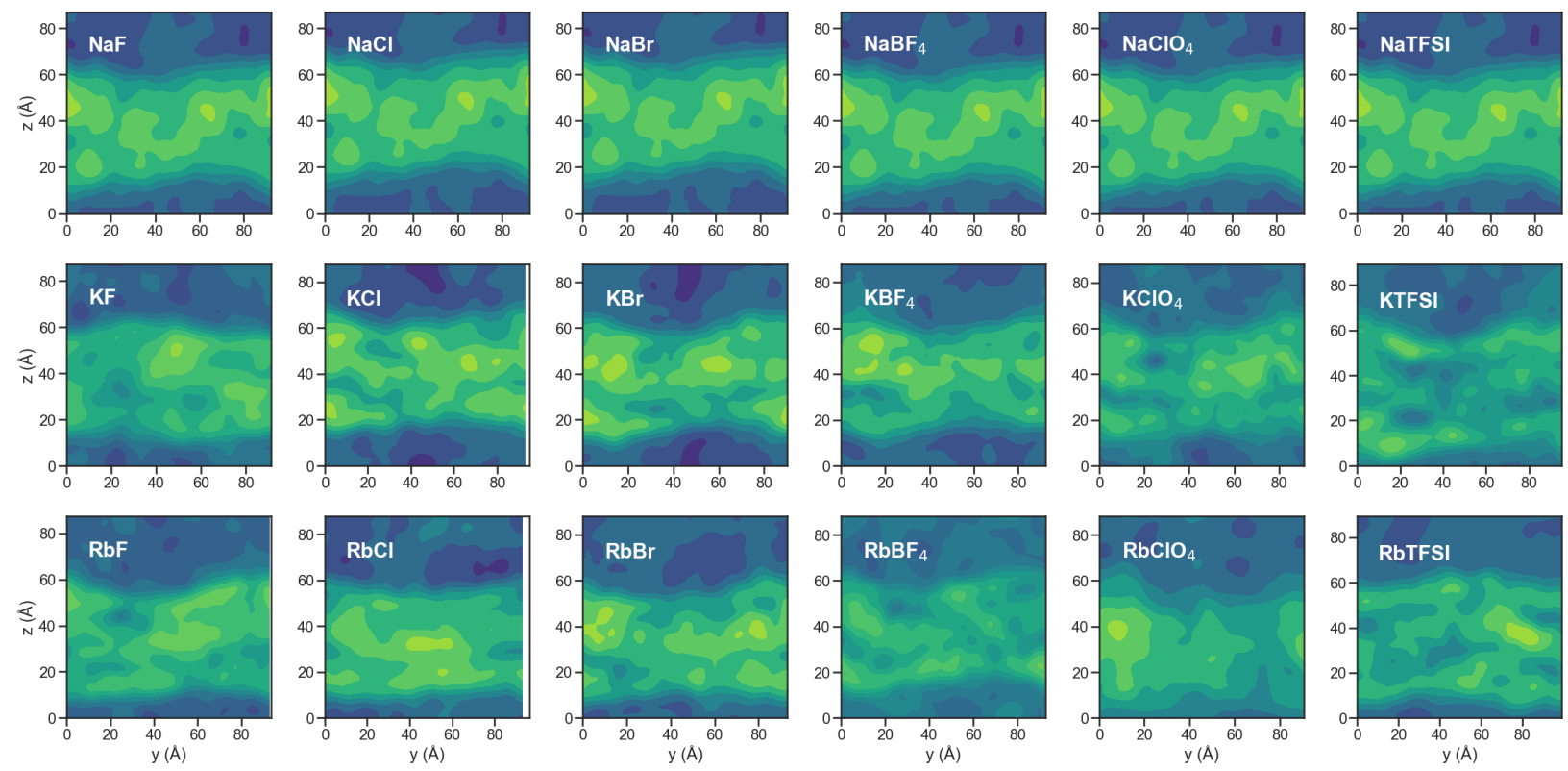

Figure S2. Electrostatic potential profile across the simulation box, for $\mathrm{Na}$ (top), $\mathrm{K}$ (middle) and $\mathrm{Rb}$ (bottom) salts. The surface plots show an average of the cross section of the electrostatic potential in the xz plane. Higher values (green-yellow) correspond to the polymer phase, while lower values (blue) correspond to the solution phase. The color scale is the same for all plots $(-20$, 20). $50 \mathrm{MD}$ snapshots were averaged to obtain the potential profiles.
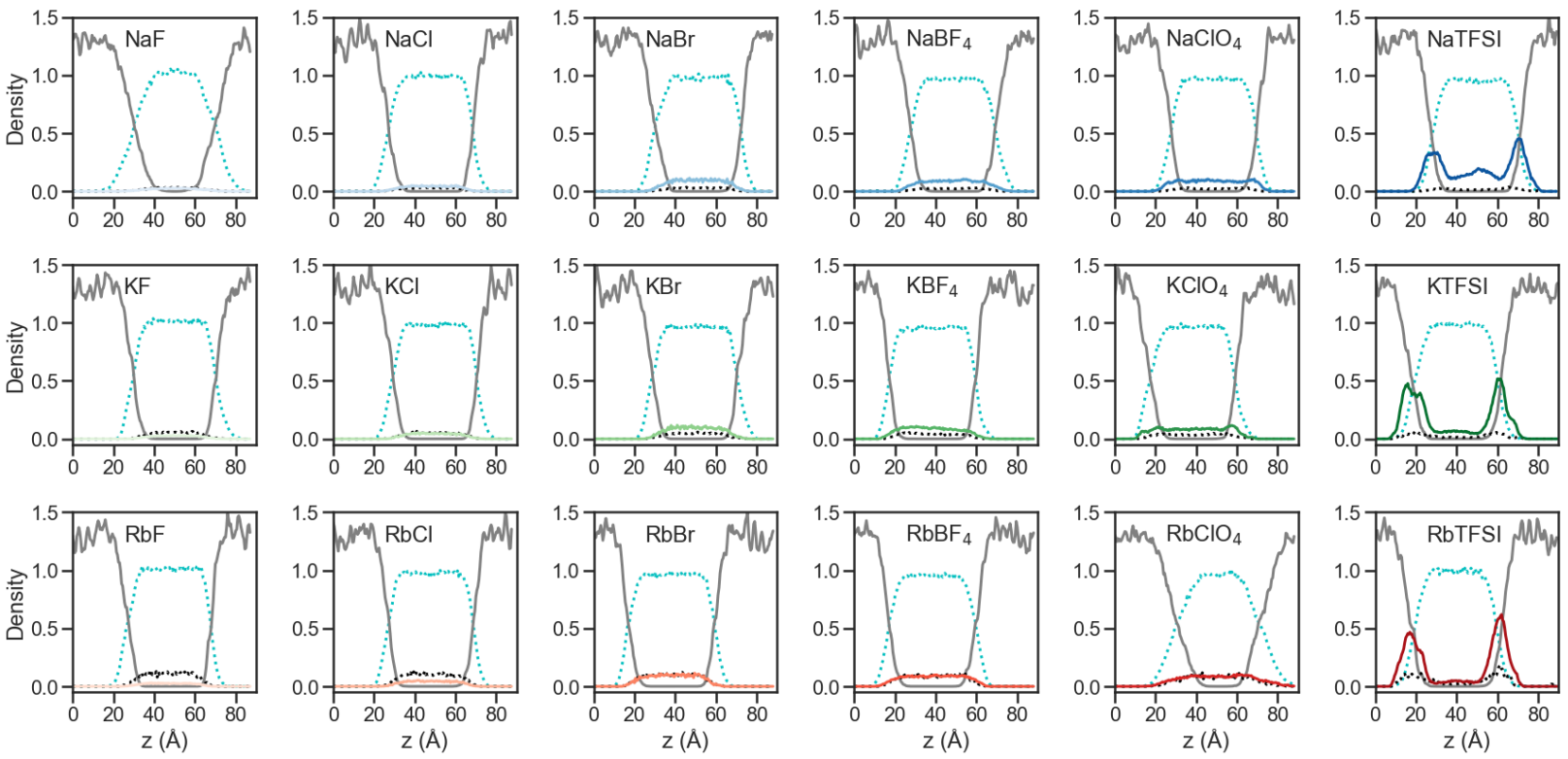

Figure S3. Density profile along the $\mathrm{z}$ axis of $\mathrm{p}(\mathrm{g} 2 \mathrm{~T}-\mathrm{TT})$ (grey line), water (cyan dots), $\mathrm{M}^{+}$ (black dots) and anions (solid lines). The water phase is wrapped to the center of the simulation box. 

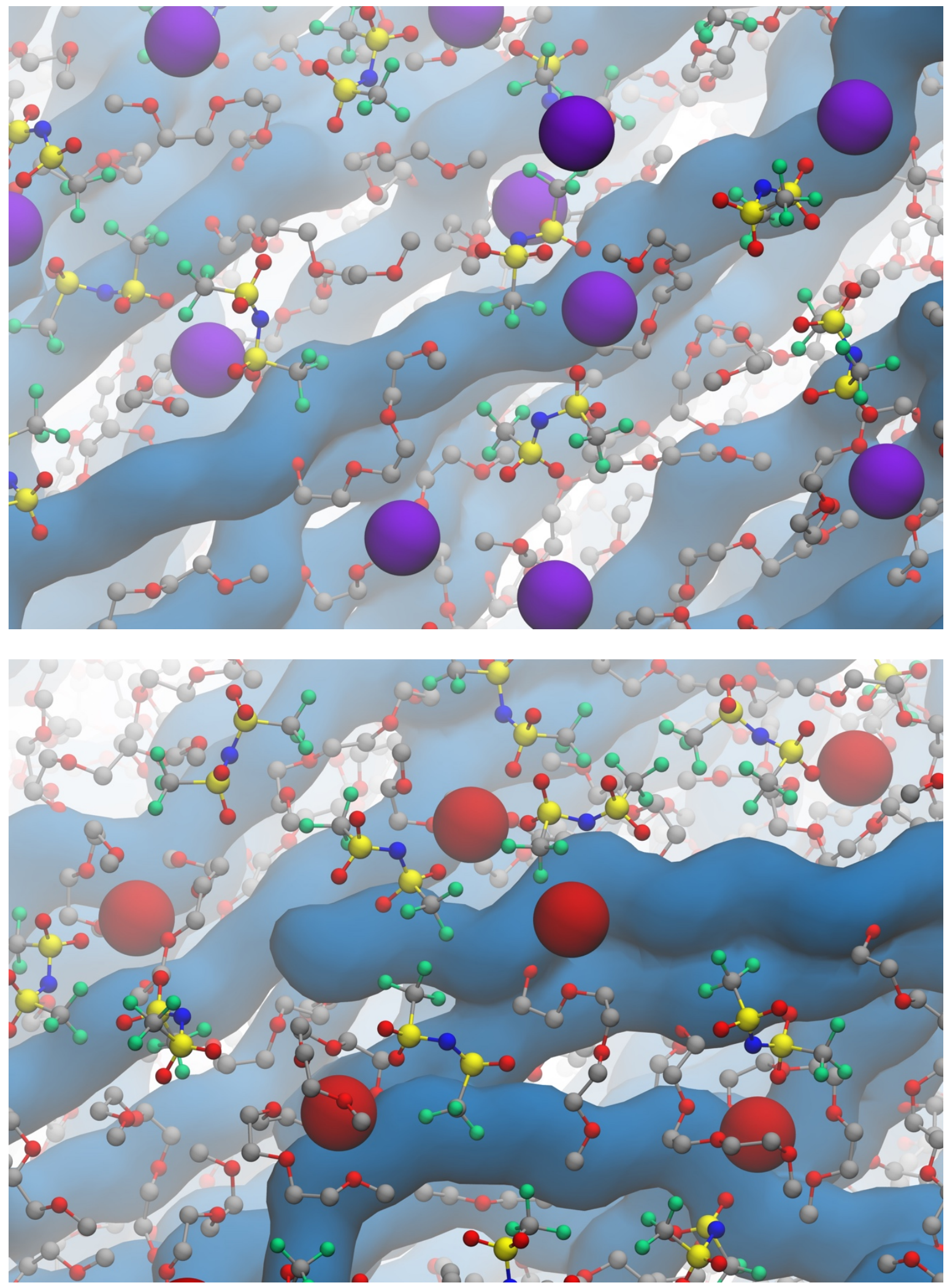

Figure S4. Snapshots from KTFSI (top) and RbTFSI MD simulations. Water molecules and hydrogen atoms are omitted; the $\mathrm{p}(\mathrm{g} 2 \mathrm{~T}-\mathrm{TT})$ backbone is represented as a blue density surface. 


\section{X-Ray Fluorescence Spectra}
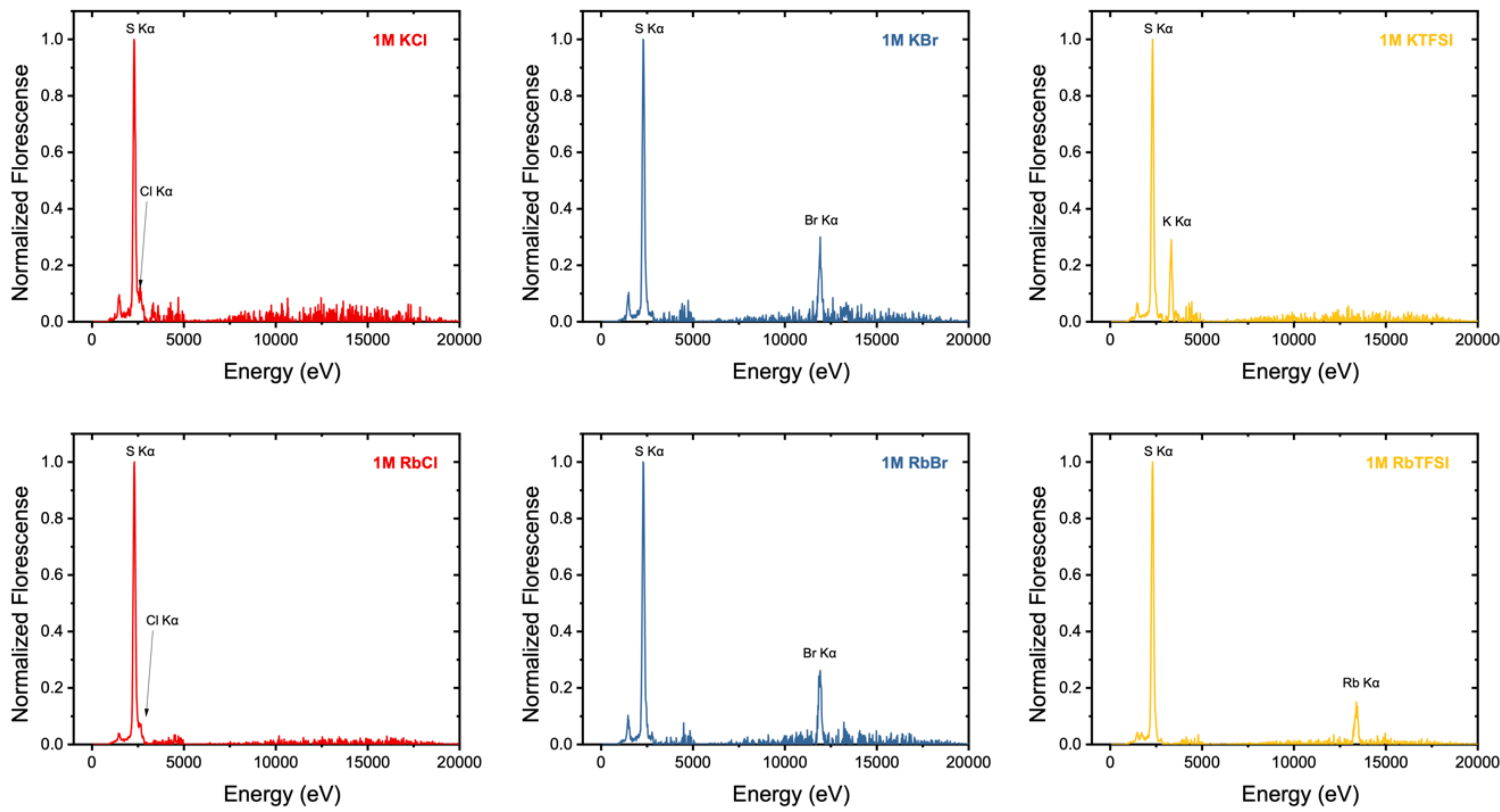

Figure S5. Individual XRF spectra of $\mathrm{p}(\mathrm{g} 2 \mathrm{~T}-\mathrm{TT})$ films ex situ exposed to $1 \mathrm{M}$ aqueous solutions of $\mathrm{KCl}, \mathrm{KBr}, \mathrm{KTFSI}, \mathrm{RbCl}, \mathrm{RbBr}$ and $\mathrm{RbTFSI}$, each normalized to $\mathrm{S} \mathrm{K} \alpha$ fluorescence.

\section{Synthetic Details}

The TFSI salts were synthesized using a modified literature procedure ${ }^{1}$. Briefly, HTFSI (25 g, $0.089 \mathrm{~mol}, 2.05 \mathrm{eq})$ was dissolved in methanol $(50 \mathrm{~mL})$ and added dropwise via an addition funnel over $\sim 15$ minutes to a suspension of the appropriate carbonate ( 1 eq) suspended in methanol (300 $\mathrm{mL}$ ) cooled in an ice bath under vigorous stirring. As the reaction proceeded, the suspension slowly cleared $^{2}$ and the reaction was allowed to proceed overnight at RT. The resulting methanolic solution of the TFSI salt was filtered, and the solvent was removed via rotary evaporation, producing a colorless solid. The solid was recrystallized from methanol to give the final TFSI salts as white crystalline solids. The salt solution was neutral after recrystallization.

\footnotetext{
${ }^{1}$ Xue, L.; Padgett, C. W.; DesMarteau, D. D.; Pennington, W. T. Synthesis and Structures of Alkali Metal Salts of Bis[(Trifluoromethyl)Sulfonyllimide. Solid State Sci. 2002.

${ }^{2}$ When $\mathrm{Rb}_{2} \mathrm{CO}_{3}$ was used, the solution never fully cleared, likely due to some insoluble impurity in the commercial starting material. This was easily removed by filtration.
} 


\section{Nuclear Magnetic Resonance}

RbTFSI: ${ }^{19} \mathrm{~F}$ NMR (470 MHz; D2O): $\delta$-76.63

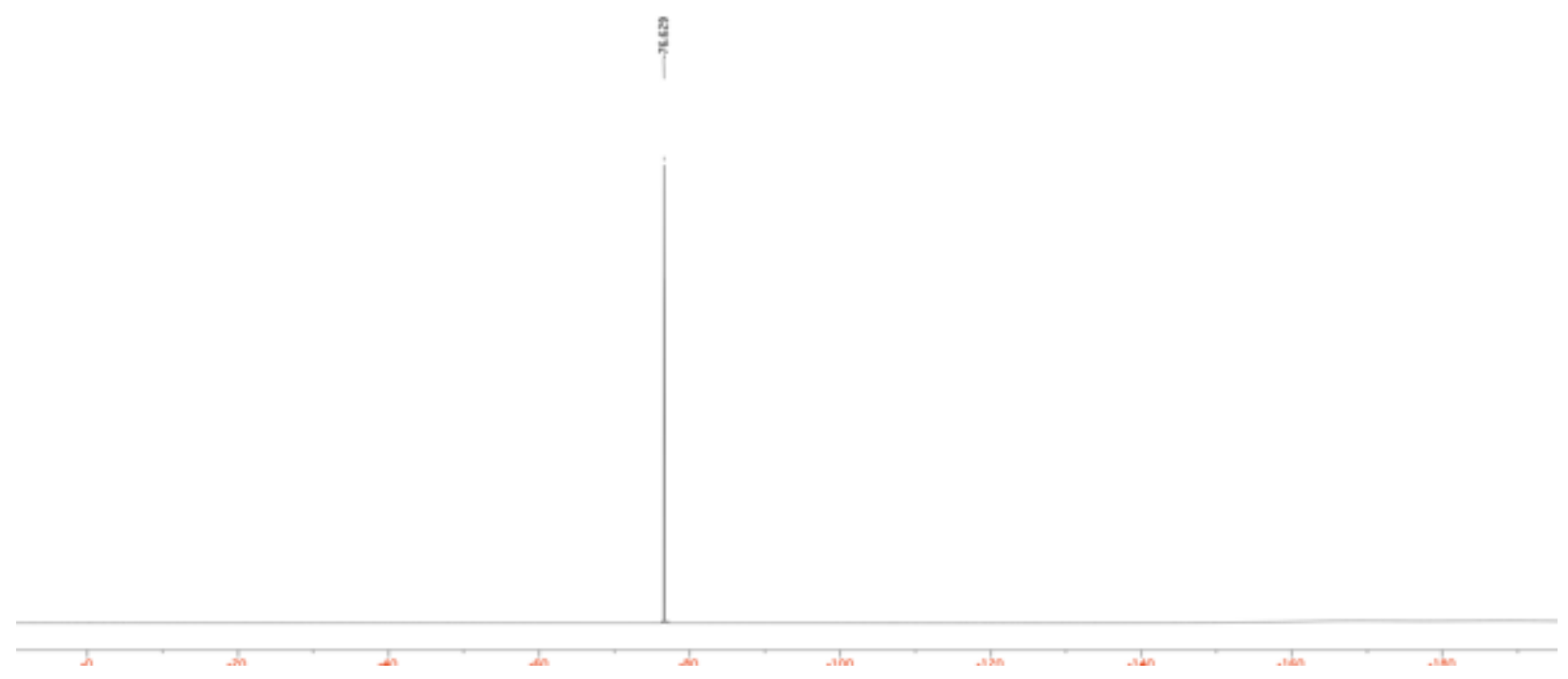

Figure S6. ${ }^{19}$ F NMR of RbTFSI.

KTFSI: ${ }^{19}$ F NMR (470 MHz; D2O): $\delta-79.18$

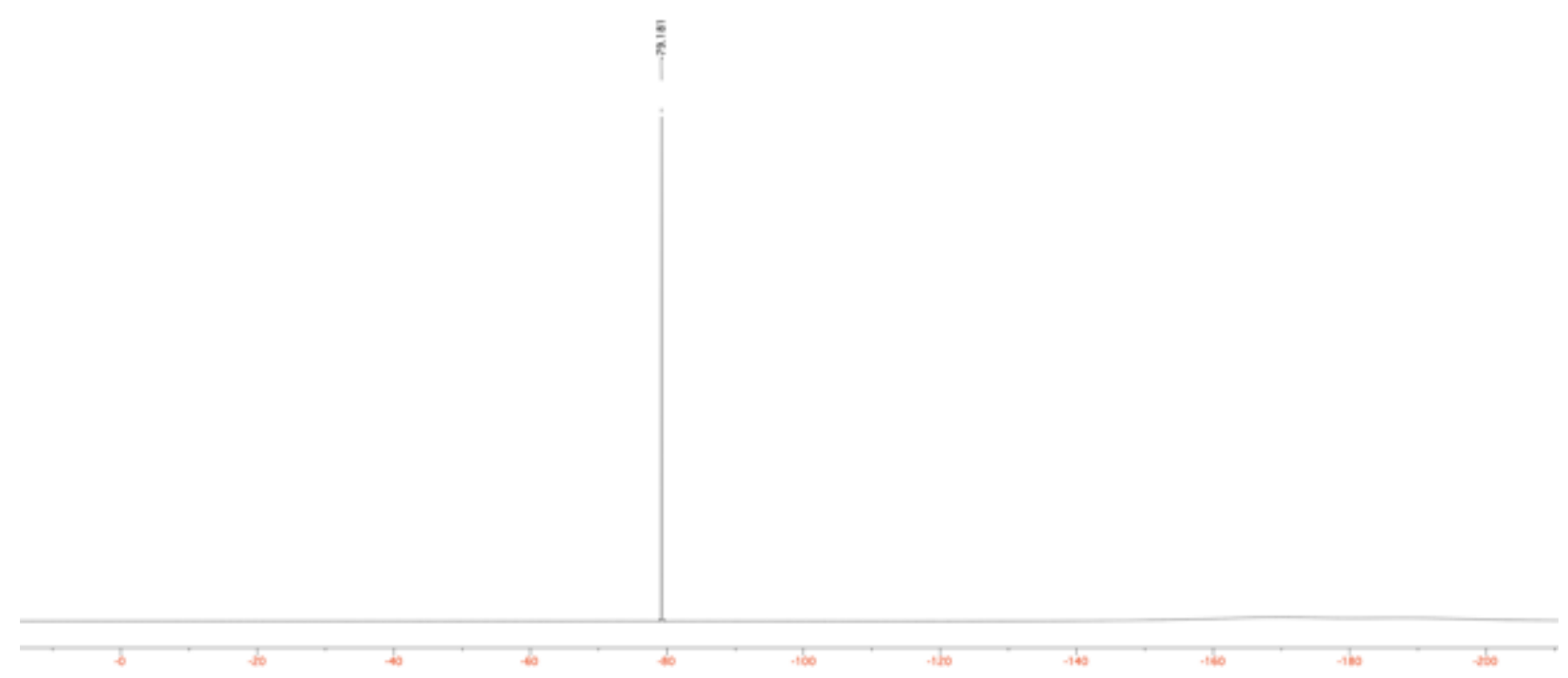

Figure S7. ${ }^{19} \mathrm{~F}$ NMR of KTFSI. 
